\title{
Einleitung.
}

Shiller war von Mambeim, wo ex jeinen treuen Freund Streidber ${ }^{1}$ ) verlajien hatte, unter den trübjten $\mathfrak{u m i t a ̈ n d e n , ~ a l s ~}$ Frembling unter irembem Manten, beforgt um bie Maápregeln, bie Der Şerzog von Württemberg gegen ben Flübatling nehmen fonnte, ängjtlich um bie Qieben in ber Seeimath, getäujht in feinen Erwartungen und ungemiß $\mathfrak{u m}$ fein ferneres Bsefdidf am 7. De= cember in Meiningen ${ }^{2}$ ) angefommen, wo er Reinmald Das erfte Mal fah und nod an bemfelben Tage nadi $\mathfrak{B a u e r b a h}$,

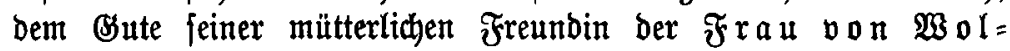
zogen ${ }^{3}$ ), ging. Reintwalb war einer ber Benigen, bie fdnell in Squiller ben aufitrebenden Bienius erfannten, wie bie fđjöne prophetifde $\mathfrak{A} \mathfrak{u} \mathfrak{f}$ eid $\mathfrak{n} \mathfrak{n} \mathfrak{g}$ aus ber exften Beit feiner $\mathfrak{B} e=$ fanntfafaft mit bem jungen Didhter, bie er in fein Tagebud ${ }^{3}$ )

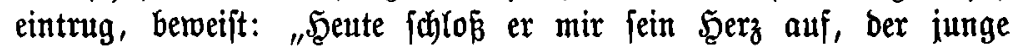
Mann - Sdjifler - ber fo früh fdon bie Sdhule bes Rebeng Durdhgemadyt, und id habe ihn würbig befunden, mein Freund zu

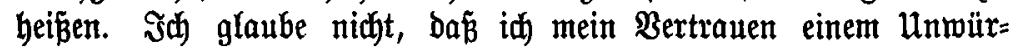

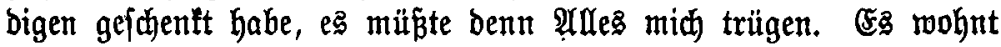

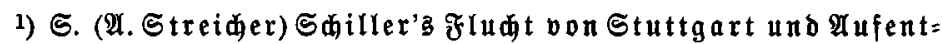
halt in Mannheim bon 1782-1785. Stuttgartu. Augaurg. 1836. 80. S. $77-81$.

2) S. Briefwediel S. 8. Anmertung 2.

3) ธ. a. a. 0 . $\subseteq$. 4 Inmertung 3.

4) S. Abenb=8eitung. 225. Donneritag, am 19. September 1839.

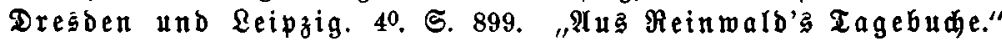
Mitgetheilt oon Rubtoig Röbler. 
ein aujerordentlider Bseift in ihm und id glaube, Deutfd)= ranb wirb einft feinen Namen mit Stolz nennen. In habe bie Funten gefehen, bie biefe vom Shicfjal umoüfterten Augen fprügn und ben reidyen Sseift erfaunt, ben fie ahnen laffen. $\left.\mathfrak{F} .{ }^{1}\right)$ ift berjelben Meinung. IUud er ahnt ben lojtbaren Schab, ben ber Steid mit feinen Sabladfen zu begraben tradjtete; aber Das Ssenie bridjt jid $\mathfrak{B a h n}$ und jollten afle Leiben ber Welt es überfluthen!" -

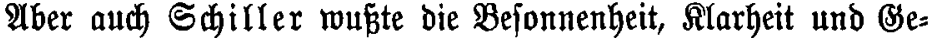
Iebrjamteit bes Freundes zu făjäben, und in biefem freundfdaftlichen Berfehr lernte $\Re$ einmald aud einen $\mathfrak{B r i e f}{ }^{2}$ ) fennen, ben Sdjiller

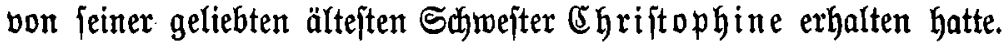

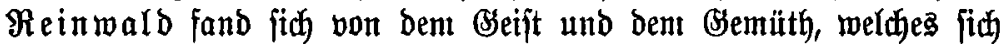

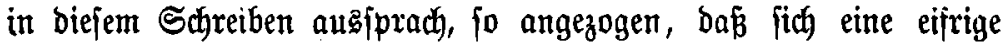
Correppondenz mit ber Sdreiberin entrictelte.

Reinmald bat, bie Shiller'fue Familie auj ber Solitude

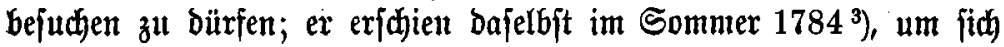
um Shriftophinens Samb za betwerben. Weber bie Eltern, nod

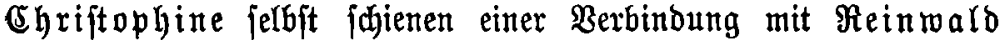
ganz abgeneigt zu fein; bemodh fam ę zu teiner enticheibenden $\mathfrak{F}_{\mathrm{r}}=$ flärung. Um Ehriftophinen, die ihren Bruder in Mannheint

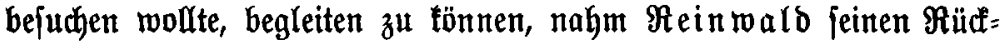
weg über bieje Stadt. Shiller war hod erireut, jeitre gefiebte Ehrift ophine in Begleitung einez werthen Freundes wiederzufehen, fühlte fich aber zugleidh unangenefnn berilihrt, als er wahrnahm,

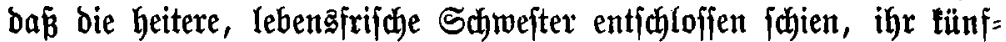
tiges Schicfial mit einem zwanzig sahre älteren Mlanne zut theilen, beffen geringe (Eintünfte und hypodjondrifde Launent wenig Freube

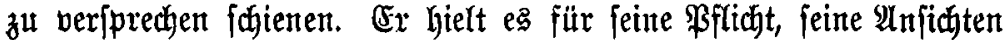

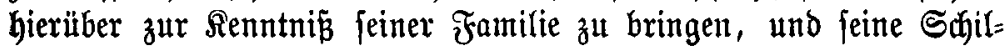
berung von $\Re$ einwald's eigenthümlichem $\mathfrak{B}$ ejen führte mirtlich einen Stillftand in ben weiteren Berbandrungen Gerbei. ${ }^{4}$ )

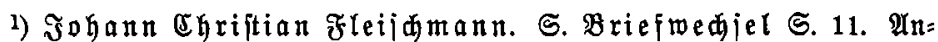
merfung 1 .

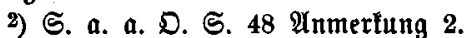

3) S. Brieftredfel S. 70 bie Ammerfung zu bem Briefe vom 13. $\mathfrak{A} u=$ guft 1784.

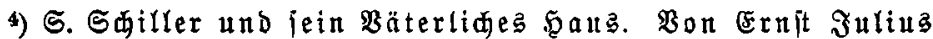
Saupe, Reipzig $1851.8^{0}$. S. 115-116. 
(Ehriftophine felbjt war fid vollfommen tlar, was fie an ihrem Theile zu thun und za laffen habe, und hatte fid bereitz mit frommer $\Re$ efignation in bie $\mathfrak{M}$ öglictłeit ergeben, $\Re$ einmald's

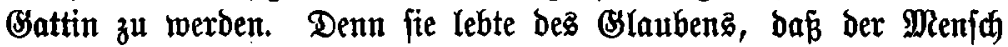
bem lieben Botte eine bejonbere Leiftung ala Beitrag zum Belt=

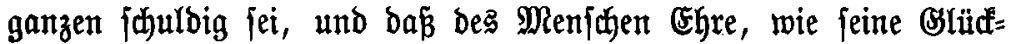
jeligfeit, barin bejtehe, zu erfennen und zu erfüllen, was Gott von ihm Bejonderes gełeiftet haben molle. Dennod) ehrte fie des $\mathfrak{B r u}=$

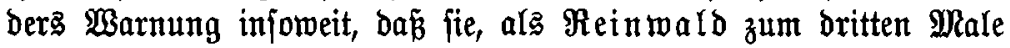

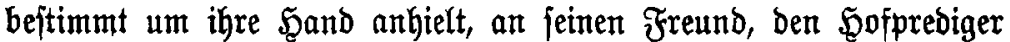
Pfranger, $\left.{ }^{1}\right)$ fajrieb und biefen um Fath fragte. $\mathfrak{B}$ franger ant= wortete ihr: aus ihren Briefen lenne er ihren anjprudjalojen und

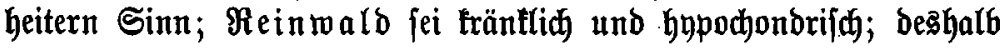
ftehe er eiman und freubenlos in ber Melt, und fie würbe, wenn fie fich entichließen fönnte, mit ihm und für ihn za leben, Blüa uns Şeiterfeit in jein einfames Dajein bringen. Dieje $\mathfrak{A}$ ntwort

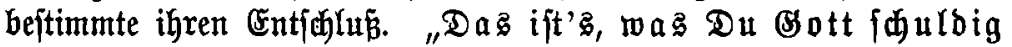

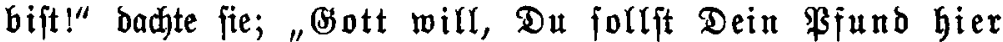
anlegen." und nun fagte fie freubig $\mathfrak{\Im a}$, und reidte $\Re$ einwald

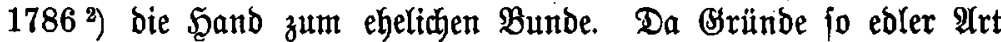
ihre Şandlungliweife beftimmt hatten, fo ift es nicht zu verwundern, baß̃ fie aud in ber Folge es nie bereute, bag Şerz ihrem Berftande und einem folchen (Satten geopfert zu haben. ${ }^{3}$ )

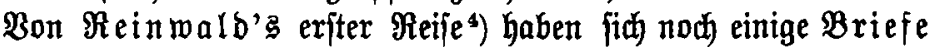
erhalten bie er - von $\mathfrak{A} \mathfrak{n} \mathbf{b} \mathfrak{b}$ ach am 18. Sulfi, brei von ber Soli $=$ tü de und von $\mathfrak{B}$ illhelmsthal (bei Frantfurt am Main) - an feinen Freund Den Šofprebiger $\mathfrak{B f r a n g e r}{ }^{5}$ ), in Meiningen, richtete und fich fpäter zur Beröffentlidung zurüroferbat, wie auz

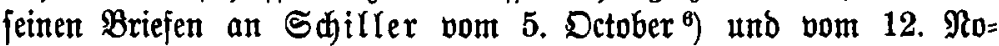
vember $1786^{7}$ ) heroorgeht. Wir theilen dieje orei Briefe von

1) S. Briefwediel S. 20 Anmerfung 4.

2) S. Briefwedfel S. 90 bie Anmerfung zu Dem Briefe vom 5. Dcto= ber 1786 und S. XL.

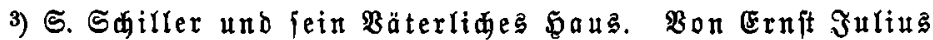
Saupe. Leipzig, 80. S. 116-117.

4) S. Briefrediel. ऽ. 70 น. 71.

5) S. a. a. D. S. 71

b) 5. a. a. D. S. 91.

7) ธ. ล. a. D. ธ. 96 . 
ber Solitübe und ein Bebet von Ehriftophine, bas biefe auf

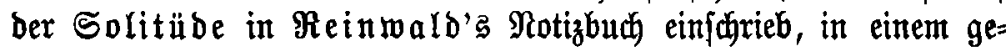
nauen $\mathfrak{A b b r u d f}$ im $\mathfrak{A} \mathfrak{n h a n g ~} \mathrm{I}^{1}$ ) mit.

Bevor wir über $\Re$ einmald weitere Radidiften geben, laffen wir hier zuerjt bas von jeiner Battin - balb nad jeinem Tode

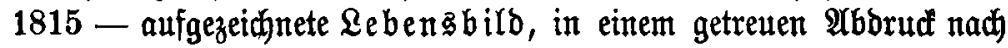
ber Driginalhanbidrift, mit einigen von uns hinzugefügten $\mathfrak{A} \mathfrak{n}=$ merfungen folgen.

1) $5 . \mathfrak{U} \mathfrak{n} \mathfrak{a n g}$. S. $261-281$. 
"Wilhelm Friebrid Sermann Reintwald ijt 1737. Den 11. $\mathfrak{A}$ ugujt in $\mathfrak{B a f u n g e n}{ }^{1}$ ) gebohren. Sein $\mathfrak{B a t e r}{ }^{2}$ ) war bajelbjt

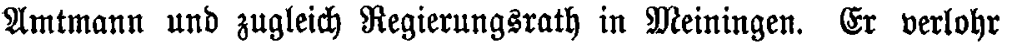
ihn fahon in feinem 14. Sahr ba er nod nidft feine Sdjuljahre beendigt hatte. Nach biefen gieng er 1753 auf bie Univerfität nach Sena, wofelbft er fich bem Stubium ber Rechte widmete und zu jeiner (Erholung feine Liebe für Didhttunjt und Sprachforfdyung aus= zubilden fuchte."

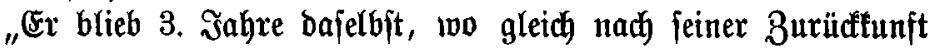
in feiner Bateritadt, ber fiebenjährige Sirieg ${ }^{3}$ ) au\$brad, burd ben feine $\mathfrak{M u t t e r}$, eine $\mathfrak{B i t t i v e}$ von 4 Sindern fehr viel verlor, und ihm felbjt bie von bem Bater nod̆ ziemlich zahlreidhe Bibliothel gänzlich geplünbert wurbe."

"Diefe ungünjtigen Umitände wirften frühe f̧hon eine miß̄= mütbige Stimtmung, und ex würbe ihr oft unterlegen fenn, wenn

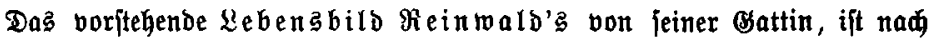
ber una vorliegenden Driginalhandjurift der Berfafferin abgedrudt, bie

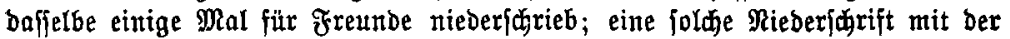
unirigen faft gleidiautend murbe in Deutiches Mufeum. Serauge:

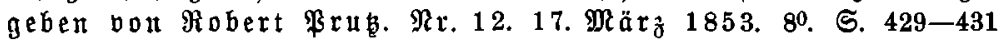

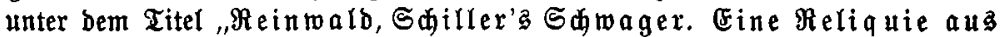
Dem Radla mitgetheilt.

1) Untweit Meiningen.

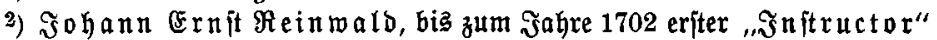

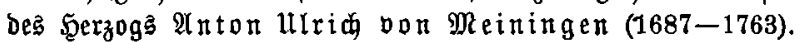

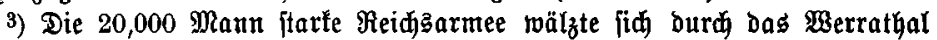
und plagte Stäbte und Dörfer. Alm 12. September 1757 zog das feer nach

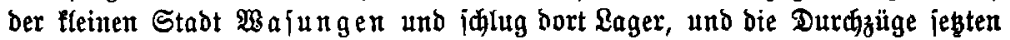
fiti bis in Den Dctober fort. 
bie Borjehung ihm nid)t in feinem verehrten Snfel, Scerrn $\mathfrak{g}$ ofrath Stieler ${ }^{1}$ ) in (Spotha, Bruber jeiner $\mathfrak{M u t t e r}_{\text {, den }}$ Berlujt feines $\mathfrak{B a}=$

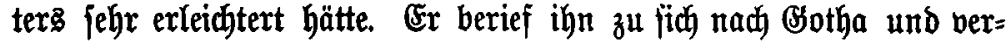

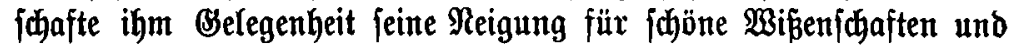
Rünite mehr auszubillben, und er hatte bas Blül von bem gefdhicf=

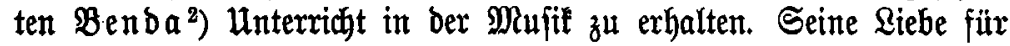
bieje Runjt jo wie bie für ßoefie und Spradfforjdung begleitete ihn Durch jein ganzes Leben und exheiterte ihm und peinen Freun= ben mandie Stunde."

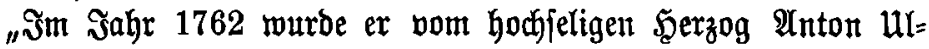

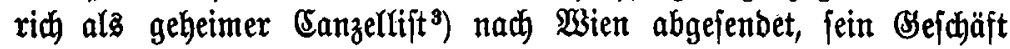
Dajelbjt wax bem \$erzog jebe $\mathfrak{B o d j e}$ Beridjt über Riteratur, Staats= jaden zc. nad Frantfurth wofelbjt fich ber Sherzog mit feiner Bse=

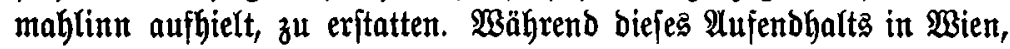
ber $1 \mathfrak{J a h r}$ nur bauerte, ftarb ber edle Fürrft, ber Bäterlich für ihn beforgt war, und ex wurbe unter bem Borwand einer beßeren Ber= forgung von bem bamaligen Minifter zurüafberufen. Sein \$flidit= gefühl, bem $\mathfrak{B a t e r l a n b e ~ f e i n e ~ D i e n j t e ~ z u ~ w i b m e n , ~ r i e f ~ i h n ~ v o n ~ j e n e n ~}$

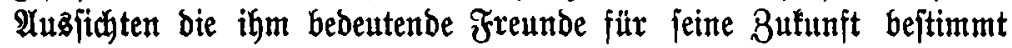
hatten zurüd, und er fehrte nad) feinem Baterlande. 䟠er wie fehr murbe er in jeinen Erroartungen für jenes Beriprechen getäujdt, ba eine ärmlide, ganz unter feinem (S)efft und Sienntnipen, nehmlid bie

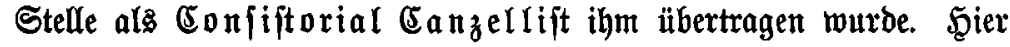

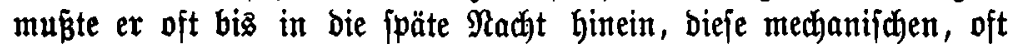
blos bie $\mathfrak{A}$ ugen anjtrengende Befdäfte verridten, beren Folge eine

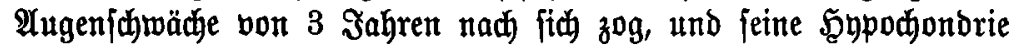

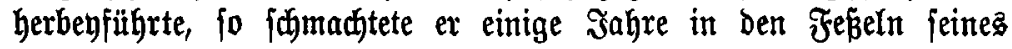

1) S. Briefwediel S. 134, 215 und 245.

2) Beorge Benda, einer ber bedeutenoften Romponiten, geb. zu Jung=

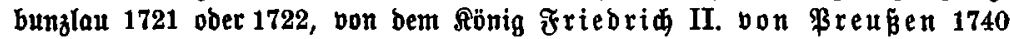
mit jeinem älteren $\mathscr{B r u b e r} \mathfrak{F} \mathfrak{r a n z}$ in $B$ erlin angeftellt, 1748 als Rapellmeifter

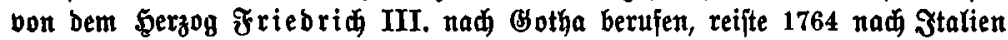

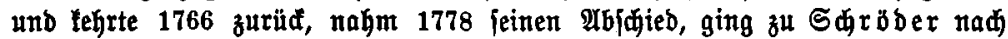
\$amburg als Mufifbirettor und hierauf nad Bien, tam aber bald wieber nad

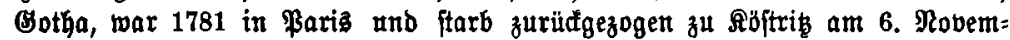
ber 1795.

3) Das ,Beitallungzbecret" ift Datirt ben 12. Dctober 1762. Rein: walb belam bie Stelle bez bajelbjt am 26. September verjtorbenen \$erzogliden „Accesfisten“ $\Re$ eppert. 
Almtes, bey oft gänz̧lidyent Mangel an ben nöthigen Rebenzbebürf= nifien wo nur bie Theilnahme inniger Freunde feinen $\mathfrak{M R t h}_{\text {erhiel= }}$ ten, bißß enblid eine hellere Bufunft fïd öfnete, unb feinem Streben

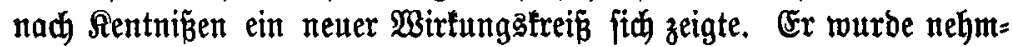
lich im sabr $1776^{1}$ ) bey herzogl. SBiblitothet ala Behülfe angeftellt und fand in biejem $\mathfrak{A}$ mte Ginreichende $\mathfrak{R a h r u n g}$ für feine $\mathfrak{\text { Biphbe= }}$ gierbe, und ob audh gleid hier mit mandhen Schmierigteiten lämpfend fo war er bod mehr als jeber Âtore geeignet jeine Liebe für jo

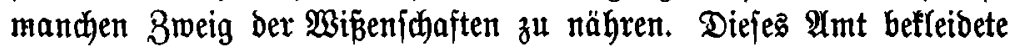

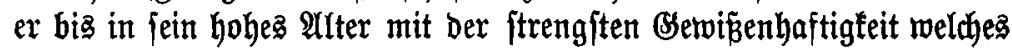

\section{1) $\mathfrak{A} \mathfrak{m} 1 . \mathfrak{\Im} \mathfrak{n} \mathfrak{i}$.}

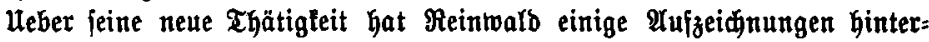

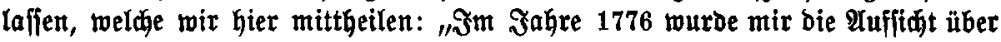

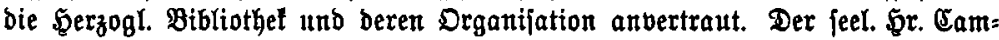
merrath $\mathfrak{M}$ oll wi hatte jie in feinen Rebenjtunden zu itellen angejangen, io weit fie aus ben Beriकlägen bon Franffurt ausgepadt war, uno ein untun= biger ober Semilitteratus bie Fortfęung übernommen."

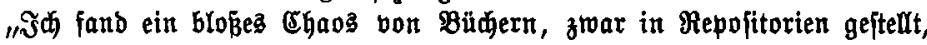
aber aus Mangel an Folivfädern oft oben zuttächji ber Deče in Duobezfädaer

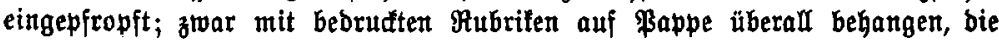

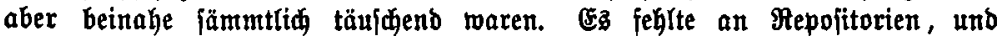
weldge madeen zu laffen, wurbe mir theils jhjwer gemadit, theils verweigert.

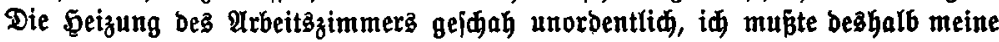

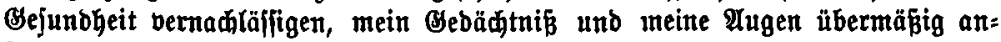

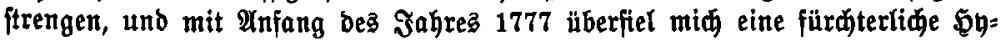
podonorie, mit anhaltendem Sdivindel begleitet, beren erjte hejtige Bufälle ben

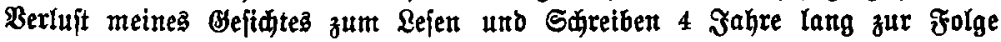

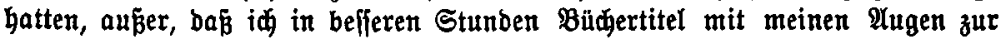
Noth aufzufaffen vermodte."

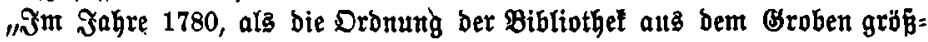

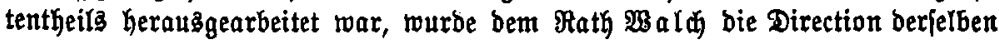
aufgetragen, ber fith bejonbers bes Meuferen berjelben antahm, und bem bie

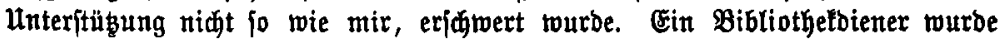
bejtellt, Tage ber Deffnung ber Bibliotbet für's \$ublifum ausెgejeģt und für regelmäp̈iges Đeizen im Winter gejorgt." Trob jeiner untergeoroneten Stel= Iung twar und blieb Reinmald bie Seele und ber gute Benius ber Bibliothet.

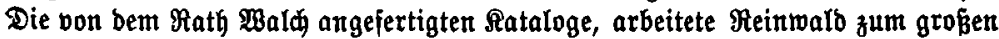

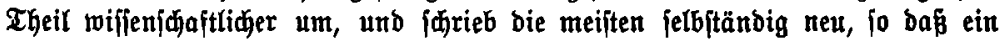

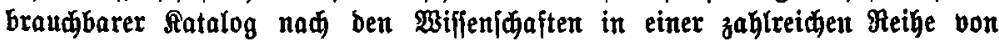
Bänden Meinwalds bauerndes Dentmal auf ber Bibliothel geblieber ijt. S. Mittheilungen aus bem Leben ber Serzoge zu Sablen Meiningen. Bon $\mathbb{R}$ ubrig Beditein. Salle, 1856. 80. S. 71-72. 
ihm feine Beitgenoß̉en bezeigen merben, bie ihn oft von minder Fiuth =

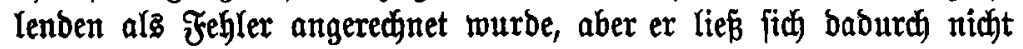

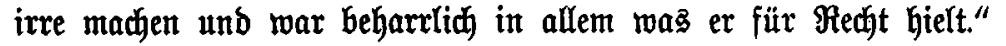

"Die meifte Beit war wipenifaftricher Unterhaltung gemibmet und er benukgte jeben 9 ugenblice den ihn fein träntlidjer Rörper

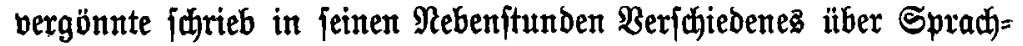
fentnike als fein Sennebergifiches \$oiotifon, und war auth Mitarbei= ter bes ulphilas. In ben lez̧teren 20 sahren war er mit ber $\mathfrak{B e}=$

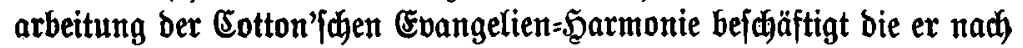
Mänchen verfaufte."

"Ein immerwährenbez Streben nach Rentniß̧en jeber $\mathfrak{A} r$ be=

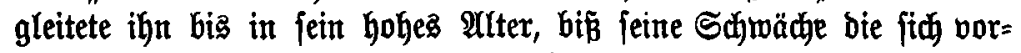
nehmlid im Bsefíd)t und sehör äuferte immer mehr zunahm und

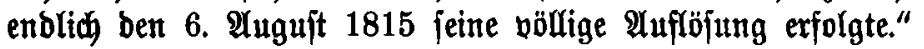

"(Er bradte fein $\mathfrak{A l t e r}$ auf 78 Jahre weniger 5 Tage und ent=

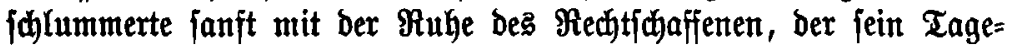
wert treu zu erfüllen bemüht wax."

"Seine Ginterlapane Biattin verlohr in ihm ben treuten Freund

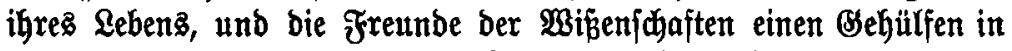

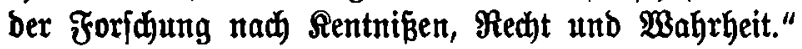

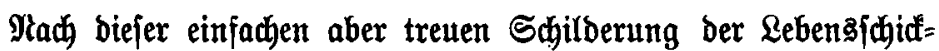
fale geben wir etne Ueberjidyt non ber jüriftjtellerifahen Thätigleit

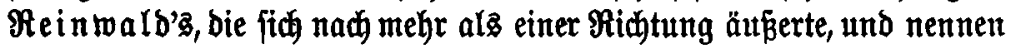
zuerit jeine poetifden Erzeugniffe, bie zmax nifft zu ben herbor= ragenojten ber Beit gehören, alle aber bie lernhafte und tüdtige (Se= finnung bes Berfaffers tragen; feine Begabung für bie Satnre und

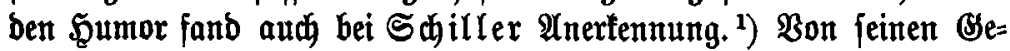
bidgten befiken wir bie beiben folgenden Sammlungen

Poetifde Briefe und fleine Eredidte. .... Meiningen, bey

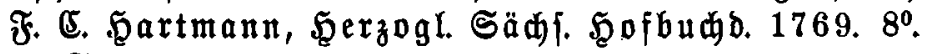
64 S.

Bon biefer erften Sammlung fennen wir nur bez Berfaffer Scanbexemplax mit vielfad,en $\mathfrak{A b a ̈ n d e r u n g e n ~ u n d ~ B e r b e f f e r u n g e n , ~}$

1) S. Briefmediel S. 81-82. 


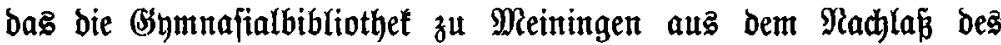
Frofeffors $\mathfrak{A} \mathfrak{u} \mathfrak{g}$ ft $\mathfrak{H e n n e b e r g e r ~ a u f b e r w a h r t . ~}$

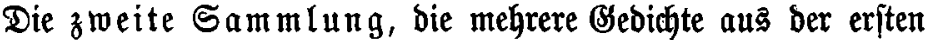
Sammlung aufnahm, exffhien unter bem Titel

Poetifbe Raunen, Erzählungen, sriefe und Mifcellaneen.

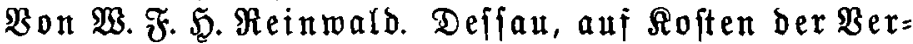

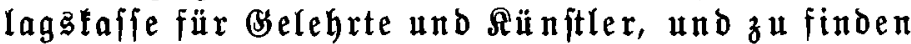

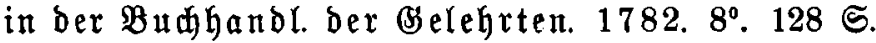

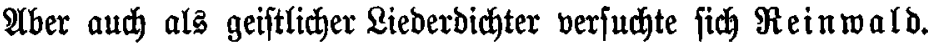

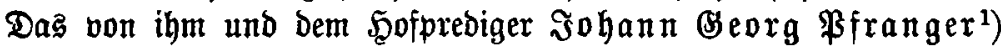
bearbeitete und nad) deffen Tode von Reinmald herauggegebene

Reues Sadfen = (Soburg = Meiningifdes Orejangbud, zur öffentliden und häuslidhen Erbaunng. Das Exemplar

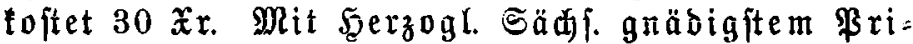
vilegio. Meiningen, gebrutt und verlegt bey $\mathfrak{B h} i=$ (ipp $\left.\mathfrak{g} \mathfrak{a r t m a n n} .1794 .8^{0} .^{2}\right)$

bringt neben bielen bon $\Re$ einwald umgearbeiteten Riebern auch fünfzehn eigene Rieber, und zwar bie Nummern 102, 428, 449, $450,514,519,546,557,565,578,593,614,615,647$ und $\mathfrak{N x} .682$, bie bas Beicjen ** tragen und von benen einige fpäter in andere Bejangbücher übergingen. ${ }^{3}$ )

$\mathfrak{A}$ wa ben hier angefühyrten \&sebidtfanmlungen geben wir im $\mathfrak{A} n \mathfrak{h a n g}$ II. eine $\mathfrak{A} u \mathfrak{s w a h l}$ nebjt einigen ungebrudten und ein= zeln gebrud ten Bedidtent.

Aluß̌er ben in bem Briefwedjfel von unz bereitz genannten

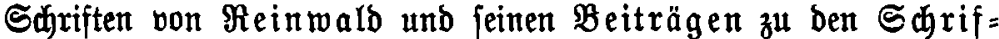

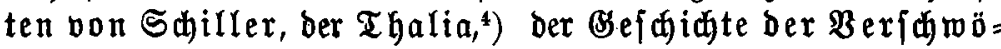

1) S. a. a. D. S. 20 Anmertung 4.

2) S. a. a. D. S. 146 Anmerfung 5 und S. 157 Anmertung 1 .

3) 5. Festschrift Zur Feier des Hoechsterfreulichen Geburtsfestes Sr. Hoheit des Herzogs Sonntag 17. December 1854 Ausgegeben von Dem Gymasium Bernhardinum in Meinin. gen. Inhalt: Meiningens Antheil an der Deutschen National. literatur. Von August Henneberger. Meiningen, 1854. 40. S.9.

1) S. Briefwediel S. 107-108. 


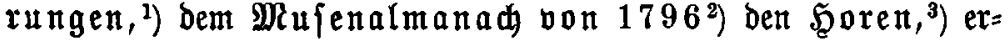

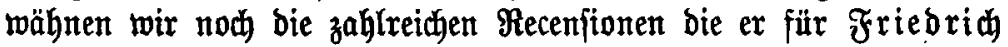
Sicolai's allgemeine beut\{de $\mathfrak{B i b l i o t h e l ~ - ~ v o n ~} 1779$ an bis zu bem Ende biejer Beitfarift 1806 - bie Bibliothel der

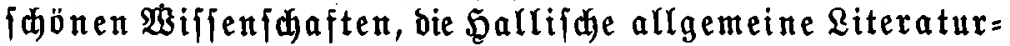

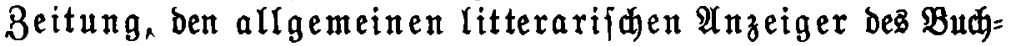
händler $\Re$ od in Reipzig und für andere Beitjafriften fabrieb und

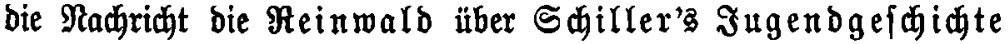
1807 beröffentlidbte, bie wir ber $\mathfrak{B o l l f t a ̈ n b i g f e i t ~ m e g e n ~ i n ~ b e n ~} \mathfrak{A} \mathfrak{n}=$ hang III. mit aufgenommen haben und wenden uns jeģt zu feinen wifienfdhaftlidben Reiftungen, bie er im Fache ber Spradjforf山ung augiührte. Die exite Heine Arbeit auf biejem Bebiet waren bie

Briefe über bie Elemente der germanifhen Sprade. 1 fter Brief. Frantfurt und Leipzig. 1776. $8^{\circ}$.

von ber wix nur ben Titel fennen. Fine Fortfekerung ift nicht er= folgt; ben Grumb hierwon gibt bex Berfaffer in ber $\mathfrak{B o r r e b e}$ bes zweiten Theile bes Soiotifon 1801 indem er jagt "bá jener

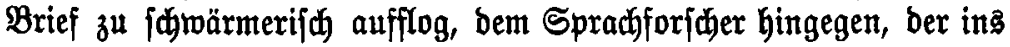
Steingebürg ber (Etymologie, wo fo viele fłon verumglüatt finb, fich wagen will, eit ruhiger unb behutjamer Sbang ziemt."

Sierauf folgte bas von feinem Landżmann Shent auz $\mathfrak{B a}=$ jungen mit großßem Sammelffeiße angelegte und bon $\Re$ einwald vollendete jebr werthoulle Mert

Sennebergifdes 3biotifon, ober Samulung ber in ber ge= fürfteten B্raffáft Senneberg gebräudliđen Jobio= tismen, mit etymologif山en $\mathfrak{A} \mathfrak{n}$ mertungen und $\mathfrak{B e r}=$ gleidung anderer alten und neuen \&ermanifden Dialefte; von $\mathfrak{B}$. F́. SE. Feinwald, Seerzogl. Sädfiff. Math und Bibliothefar in Meiningen. Das Mlterthum

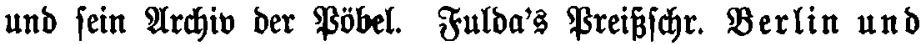
Stettin, bey Friebrid Ricolat. 1793. $8^{\circ}$. 115 S. 3wegter Theil, welder Beridtigungen, Ergänzun= gen und Bermehrungen bes Erften enthält. Boran ein

1) ร. $\mathfrak{A n h a n g . ~ S . ~ 3 0 4 - 3 2 7 . ~}$

2) S. Brieftedfer S. 161-162.

3) ธ. a. a. D. S. $172-189$. 
Berfud über bie fämmtliden Bermanifden $\mathfrak{S a u p t =}$ bialefte und einige unterfheibung und am Ende ein Berzeidnip von Bolfziörtern bes mittlern Frantens. $\mathfrak{B}$ on $\mathfrak{B}$. F. S. Feintwald, . . 1801. 171 S. u. 1 Bl. Sdyreib= und Drudfehler.

Das von bem Berfaffer an die Bibliothet deș Błymnaftum $\mathfrak{B e r n =}$ harbinum zu Meiningen zum andenfen verehrte Eremplar hiervon,

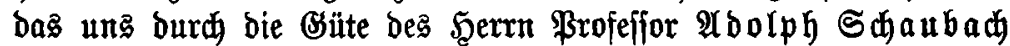
bajelbjt mitgetheilt wurbe, enthält nachjtehende eigenbändige çin= zeidnung:

Funfzigmahl hat um bie Sonne fin nun ber Grob= ball geidiwungen

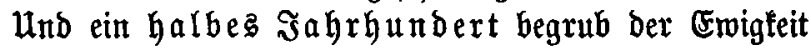
abgrumb,

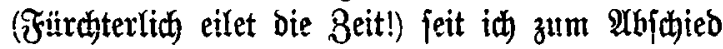
bie Şand Dir

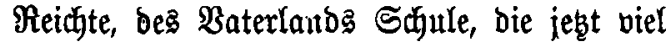
herrficter blühet

$\mathfrak{U}_{\mathfrak{n} \delta}$ viel Sünger indę̧ zu würoigen Lèhrern gebilbet!

Blüh nod ferner im Segen, um nidat nux Morte zu Yehren;

Lehr' audh Thaten: Das $\mathfrak{B a n d}$, das Menichen mit Menid)en verbintot,

Unauflöabar ber (sier nađ Serwinn und niebri= ger Selbjtiudt! -

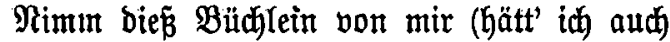
minder ber Borte

Mehr ber Thaten gejammlet!) und benfe noch mein wenn id Staub bin!

(Sefdrieben um Oftern $\mathfrak{W}$. F. $\mathfrak{H}$. Beinmald. 1801.

Aud bei Der Şerausgabe bez $\mathfrak{u l p h i l a s ~ w a r ~ R e i n n a l d ~ b e = ~}$ theiligt, ber unter bem Titel erfdien:

Ulfilas Gothische Bibelübersetzung die älteste Germanische Urkunde nach Ihre'ns Text, mit einer 
grammatisch-wörtlichen Lateinischen Uebersetzung zwischen den Zeilen, samt einer Sprachlehre und einem Glossar, ausgearbeitet von Friedrich Karl Fulda weiland Pfarrer in Ensingen im Wirtembergischen das Glossar umgearbeitet von W. F. H. Reinwald Herzogl. Sächs. Rath und Ober-Bibliothekar in Meiningen ... herausgegeben von Johann Christian Zahn Prediger zu Delitz an der Saale bei Weifsenfels in Sachsen auf Kosten des Herausgebers Weifsenfels gedruckt bei Johann Friedrich Leyckam und in Commission bei Joh. Ambr. Barth in Leipzig. 1805. $\left.4^{0} .^{1}\right)$

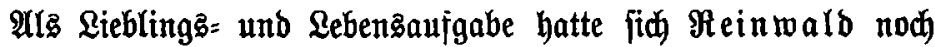

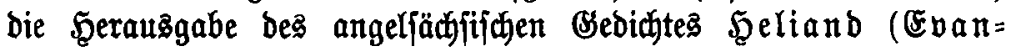
geliengarmonie) nebjt Gloffar und (Srammatil geftellt. Er erwarb hierzat eine $2 \mathfrak{b}$ fhrift von biefem Cobex bon bem \&ehrer und Lettor ber franzäjifiden Spradje am Sơnmafium zu Bamberg $\mathfrak{B}$.

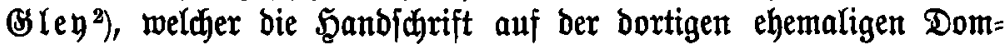
ftift=Bibliothef, als Paraphrasis evángelica im Ratalog bezeidntet, auffand, und in ihr benfelben Coder ertannte, ben Seictes in jeinem Thesaurus linguarum septentrionalium zuerft erwähnt, und $\mathfrak{F r a g}=$ mente baraus mitgetheilt hatte, und von bem fich eine Şandjärift aud in Lonbon in ber um 1602 gegründeten Bibliotheca Cottoniana befindet. Bley nahm eine $\mathfrak{A b}$ hhrift bon Dem Samberger

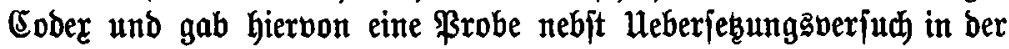
Beilage ber $\mathfrak{B a m b e r g e r} 8$ eitung von 1799 Sr. 6 S. 2123, Nir. 13 S. 49-51 und in $\mathfrak{N r}, 20$ S. 77, bie in bem $\mathfrak{L e i p}=$ ziger allgemeinen litterarifden $\mathfrak{A n z}_{\mathrm{z}}$ eiger von $\Re$ od von

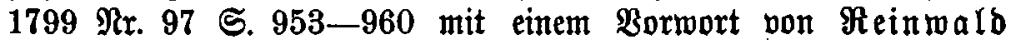
wieberholt wurben und bald barauf bon ihm nod in bemfelben $\mathfrak{s a h x}=$ gang ber genannten Beitjarift $\mathfrak{N}$. 175 S. 1737-1744 eine ", $\mathfrak{B e}=$

1) S. Briefwediel S. 231 und 245.

2) S. Sthiller'g Beziehungen S. 308 unb Mittheilungen aug bem Qeben ber Serzoge zu Sadien Meiningen und beren Bezie=

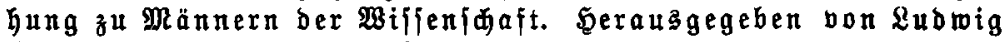
Bedftein. Galle, 1856 . 80. ऽ. 76-77. 


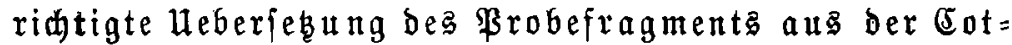
tonifaen (Eongelienharmonie" eridien. ")

(Sley theilte hierauf eine genaue $\mathfrak{A b j}$ brift bes Cober an $\Re$ ein = wal b mit, weil bie Şandidriften ber Bamberger Bibliothef in bie

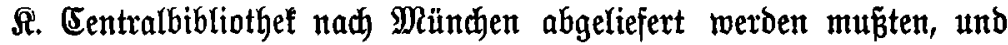
Iescterer wandte nun allen Ffleiß̄ baran, bas Bsanze in gejpaltenen Columnen mit gegenüberjtehender oeutjaer Heberjęung auşzurbei= ten unb nieberzufatreiben. (Er hatte aber nidft bie Freutbe fein müh $=$ james Merf jelbjţtändig erfheinen zu jehen, und verfaufte baher feit Manufcript nad Mündyen, wo es von Schmeller 1830 bei beffen Serauzgabe bes beliand benukt murbe.

Die ganze literarifhe $\mathfrak{A n g e l e g e n h e i t ~ u ̈ b e r ~ d i e ~ S c e r a u g a g a b e ~ d e s ~}$ Seliand finbet ber Spradyforjder in ber Şallifden allgemeinen Qitteratur=

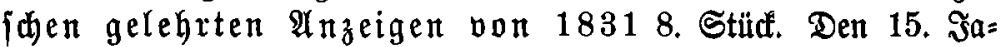
nuar, in ber SRecenfion des S๙ Brimm.

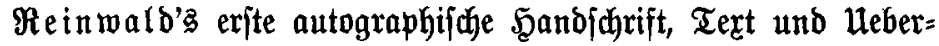
febung, im untern Rande burdjweg [pradhlid gloffirt, werehrte $\Re$ ein = walb's Wittwe an $\& \mathfrak{l} b \mathfrak{w g}$ Bed ftein in Meiningen, bem wir

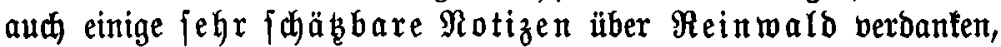
weldye ex gropentheils nod von Reinwald's Wittwe empfangen hatte, bie feine von uns fofon einige mal angejührten Mitthei= Iungen aus bem Reben ber Serzoge za Sadjen=Meiningen enthalten.

Noch haben wir über Den \&ebenggang von Reinwald's Bat=

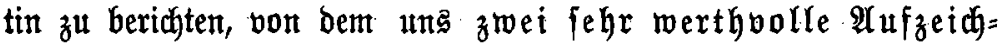
nungen unb zwar von Bexfaffern, weldye ber Beremigten im Leben fehr nahe geftanden haben, vorliegen, bie wir hier in einem $\mathfrak{A}$ u'gzuge mittheilen. Die erjte erjatien unter bem Titel $3 \ddot{u} g e$ aus bem

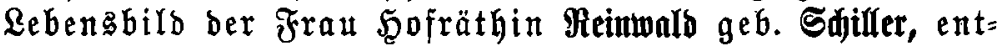
worfen von Dr. Adermann, Dberhofprebiger. Der Ertrag zu einem wohlthätigen 8 wed im Sinne ber Bollendeten. Mieiningen 1847. 80. Die ziveite ijt ber Metrolog von Shil= ler's Sdyefter von $\mathfrak{B}$. A. Baffow in Blätter für litera=

1) S. Briefmediel S. 252 . 
rifáe Unterhaltung. SRittwoh, $\mathfrak{R r}$. 265. 22. September 1847. S. $1059-1060$.

(Elifabeth Shriftophine Frieberife war baz ältejte Ritto ber Sdyillexidyen Familie; jie wax zmei Jahre vor ihrem Bruder Frit in Marbac geboren am 4. September 1757. ${ }^{1}$ ) Die (Sejdywifter

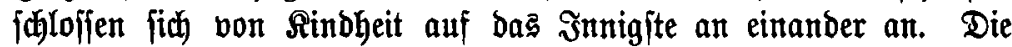

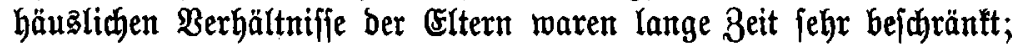
Der Bater Sohann Eajpar biente als württembergifder Dffizier, uno war gering bejolbet; bie Mutter hatte ihrem Mann lein Bermögen

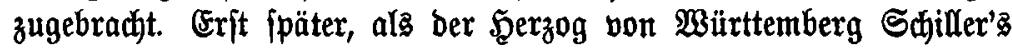
Bater auf bie Solitübe bei Stuttgart berief, um bajelbft eine Baum= ¡ăule für bas ganze Sand anzulegen, fam bie Framilie allmälig zu einigem Bohljtand.

Seier auf ber Solitübe verlebte (5hriftophine froke Tage; bas nahe Stuttgart bot mandje gejellige Freube; mit bem $\mathfrak{B r u b e r , ~ b e r ~}$

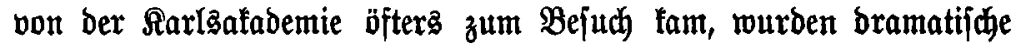

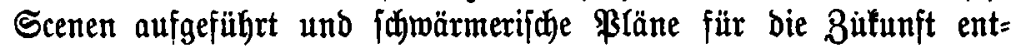

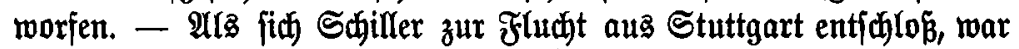
bie Shywejter feine Bertraute, bie bis an ihr Lebensende mit einer unbejabreiblidfen Innigfeit das $\mathfrak{A n b e n t e n ~ b e s ~ f r i ̈ h ~ v e r l o r e n e n ~ B r u = ~}$ bers berwahrte.

(5hrift ophinens Befanntidaft mit $\Re$ einwalb haben wir idon

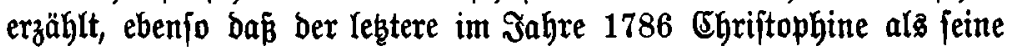
Battin nad Meiningen führte.

Reinwald hatte bei vielen trefflichen und adhtungsiwerthen ctigen=

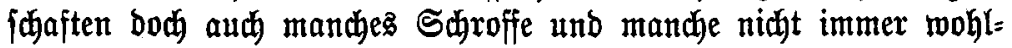
thuende Eigenthyümlidfeit; fo Gatte feine Battin Mancherlei zu er= tragen und burchzufämpfen, aber bie fittliche Bebiegentheit, ber innere geiftige Reidythum und bie jidi ftets gleid bleibende Bseduld und Seiterfeit ber trefflidjen Frau lię fie aud in ihrer finberlojen Ehe

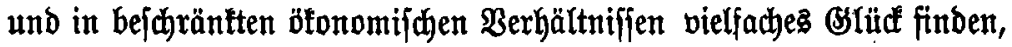
unb in turzer Beit erwarb fie fid zahlreidhe treute Freunbe und Freun=

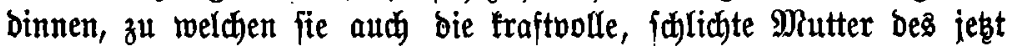
regierenden Şerzoga ${ }^{2}$ ) von Sadjen-Meiningen zählen burfte. Nadh

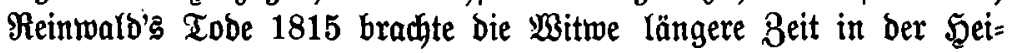

1) S. Şitrer's Beziehungen. S. 15.

2) S. Briefrediel S. 88. 
mat, vornehmlid in Marbad) zu, von wo fie auch mit einer Freun= bin einen Theil ber Sameiz bereifte; aber freundfdaftlidje Berbin= bungen und öfonomifhe Rücfichten führten fie wieber nad Mei= ningen zurüá, wo jie ein jeltenes âter in ben glürflidjiten uno fđönften $\mathfrak{B e r h a ̈ l t n i f f e n ~ e r r e i d h t ~ h a t . ~} \Re$ ux noch felten wurbe biez ruhige Leben burch fleine Reijen, z. B. nady Ruboljtabt, wo Sdjil= Ier's ältefle Todyter ${ }^{1}$ ) lebt, unterbrochen.

Arle ihre geiftigen und törperlichen Sräfte blieben ihr bis zum

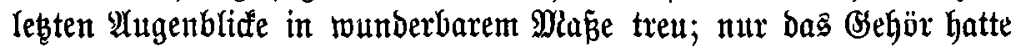
in ben lek̨ten Jahren abgenommen, bod verftändigte fie fid mit $\mathfrak{B e}=$ fannten und Berreundeten immer nod ohne befondere Miühe. Bor etwa zwei Jahren war fie an ber Brippe heftig ertranlt, fie felbft

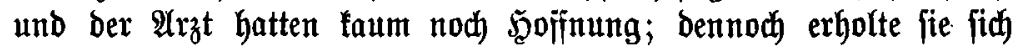
und war feitbem wo möglid nod jrifher und lebens̄voller als vor=

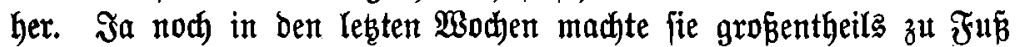
Spaziergänge nadh iajönen hodjgelegenen Punften bex untgeyent, bie fie jeit vielen Эahren nicht gejehen hatte. Sehr jelten mag Jemand in ihrem 2 llter bes Lebents fich jo erfreut, es jo heiter ge= noffen haben wie Ehriftophine Reinwald, und zugleid fo in jebem $\mathfrak{A}$ ugenbliffe zum $\mathfrak{A b j}$ bied bereit getwejen fein wie fie. "Miein Tobestag ift mir lieber als mein Beburtstag", antwortete fie, als türzłlid ihr naher neunzigiter Beburtętag erwähnt waroe. Sie äuperte wieberholt, daß̃ fie jeden Tag als ein munderbares Bnaben= gejdent Bottes betradjte, und da wenn fie nidit jeben berfelben möglicfit zu nuben, eines jeben ber= felbèn fid möglidjit zu erfreuen trachte. Sie müffe fich immer den ganzen Inhalt ihre \&ebeng zurüfrufen, um zu begreijen wie alt fie

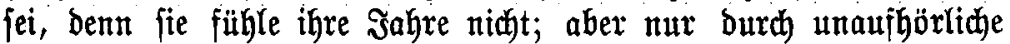
Thätigłeit tönne jie fich am $\mathfrak{Q} e b e n$ erhalten. Deshalb hatte fie immer eine ganze Reibe von $\mathfrak{A r b e i t e n ~ z u r ~ S a n o , ~ m i t ~ b e n e n ~ f i e ~ f i t h ~} \mathfrak{a} b=$

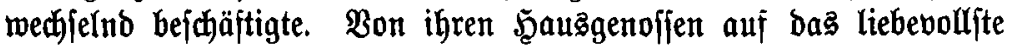
gepflegt, nahm fie bod nur wenige Dienftleiftungen an: fie fegte bis ans Ende ihre Stube felbit, fie madfte ihr Bett felbft, nidat aus Noth, fonbern um ber Befdüftigung willen; fie verfertigte fid ben

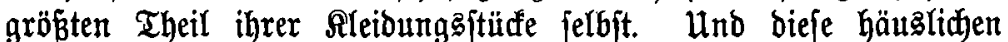
Arbeiten medbelten mit lünftlerifđen uno geiftigen. Bon frühauf

1) Saroline SEenriette $\mathfrak{Q}$ uife. S. a. a. D. S. 6. 7. 220,238 u. 239. 
eine eifrige und funftfertige Malerin übte fie biefe $\mathfrak{R u n f t}$ nod) an ihrem leksten Lebenstage. Sm Frühlinge 1845 zeigte fie mix einen

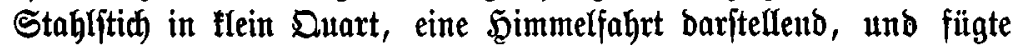
hinzu: "Das joll meine $\mathfrak{A r b e i t}$ für biejen Sommer fein, bieş Blatt zu copiren; es foll aber brei bis vier mal grö̈ß̈er werben, benn ba= bei lernt man am meiften!" Jm Şerbjte war bie Sreidezeidnung größstentheile vollendet. Mit bejonderer Borfiebe aber malte fie in Bafjerfartben Blumen und Frühte nach Der Natur; ein foldhes un= vollendet gebliebene Blatt bejüäftigte jie in ihren lebsten Tagen. Auch mit ber neuern und neuejten Riteratur exhielt jie jich fortwäh)=

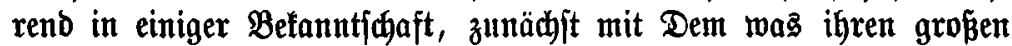
Bruber betraf, wie fie z. B. an $\mathfrak{\Re u r}$ ” "Sdirler'z Seimatz=

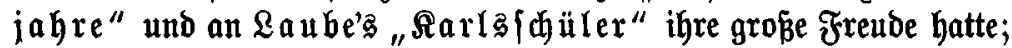
bod) wollte fie bie Darftellung ber Yeętern nidyt fehen, fie fürchtete das lebenbige Anbenfen za trüben, wenn fie den $\mathfrak{B r u b e r}$ burd) einen andern fremben Menfchen bargejtellt jähe. Ałber auch andere Sadrif= ten zu Iejen war ihr fortwährendee Bedürfni胍. So hatte fie vor einigen sahren ein einzelnes Sebidgt von Ehamifjo zur Scano be=

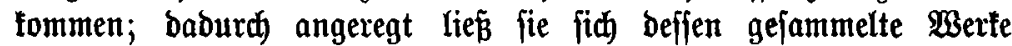
bringen, las fie fajt ganz burch und bezeidhnete bie Eigenthümlidbleit bes Didters, weldhe ihren heitern Sinn freilidh nidht burdhweg an= [prad, mit flaren und faljarfen Morten. $\mathfrak{B}$ enige Tage vor ihrem Tobe hörte fie mit gropem Sntereffe eine Mittheilung über $\mathfrak{S a ̈ n l e ' s ~}$

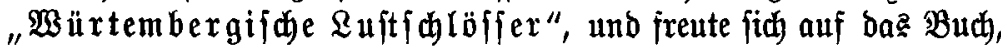
weldjes jo viele heimlide crinnerungen für jie enthielt; boch hat jie es

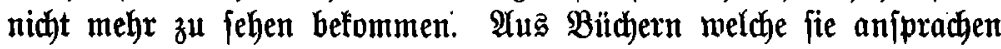

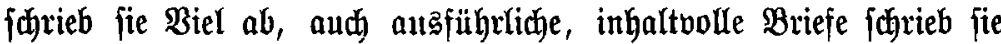
nicht eben felten in fejten, Deutlichen Bügen.

Mit groperer Borliebe Ging fie an ihrer Seimat und ihrer Jugento= zeit, bon ber fie mit feltener Rebenbigfeit und mit ftets fith fteigern=

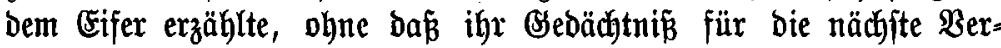
gangenheit gejdhwächt gewejen wäre. Bor łaum zmei Jahren entwar $f$ fie mir bie Yebendigfte Sdjilberung Der Solitude, und zeidnete wäh $=$ rend des Sprecteng einen überfithtlichen Brunbrip berjelben und ihrer Ulmgebungen mit Dem Bleiftift nieser. Cinen Beweiz der innern sugend und Rindlichleit bic fie fich berwahyt mag audh fol= genbe Aeukerung abgeben. Sie erzährte mix einmal $\mathfrak{B i e l}$ von ifrem

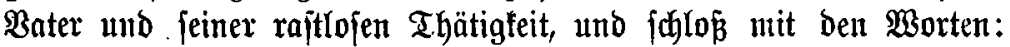


"(Er war ein ganz vortrefflicher $\mathfrak{M a n n}$; was idf aud unternebmen mag, idf frage mid immer nod zuerit, ob er es aud billigen mürde."

Eine tief religiöje driftlide Befinmung, bie fin befonders in großßem Intereffe für geiftlidge Didftungen und für bas Miffitons=

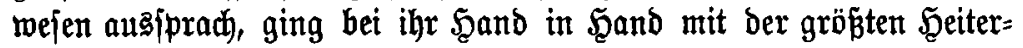
feit, bie fith nicht felten wie bei einem jungen Mäbchen in dem her $z=$ lidjiften $\mathcal{Q} a$ den und mandjem treffenden Sherz tund gab. Durd und burd aber war fie einfach, natürlich und wahr.

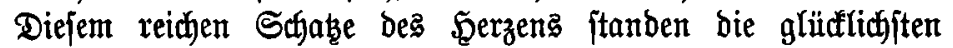
äupern Berhältniffe zur Seite! ATfer öfonomifinen Sorgen war fie

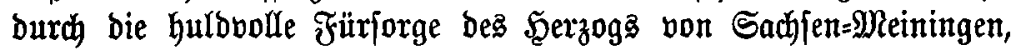

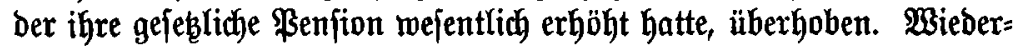
holt bot ihr berjelbe Frürit, boten ihr andere hohe Bsönneriment eine weitere Steigerung ihres (Einfommens an; fie lehnte es bantent ab,

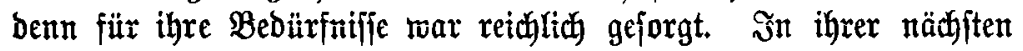
umgebung fand fie bie liebebollfte und jorgiamjte Pflege, an weldher (id) Schiller's jüngite Todhter, Emilie Freifrau vou (s)leiden= $\mathfrak{R} \mathfrak{u} \mathfrak{\beta} \mathfrak{w} \mathfrak{x} \mathfrak{m}$, und Deren Bsemahl bei ihrem häufigen $\mathfrak{A} u$ fenthalte in Meiningen auf bie jü̈nfte uno zartefte $\mathfrak{B s e i f e}$ betheiligten. $\mathfrak{B o l}_{0}$ nur felten berging ein $\mathfrak{T a g}$ an bem ber Greifin nidit burch) (Ein= heimifhe ober Frembe Belegenheit za Kebendiger Unterhaltung ge= boten wurbe, wie fie fie fo fehr liebte. Samentlich waren ihr bie Gäufigen Bejuthe Der edeln F̧erzogin und des Erbprinzen von Sahfen= Meiningen ftets eine Duelle großer Freube; aud andere fürftliche ßerjonen: Die verwitmete $\Re$ önigin von \&rojbritan= nien, bie Serzogin Bernharb und ber Erbgropherzog oon Weimar fuchten Sdjifler's Sdymefter auf. Mit herzlicher Freube, aber ohne Eitelfeit jprach fie von foldhen Bejuchen, Durch bie fie fich

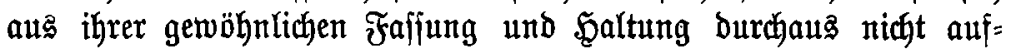

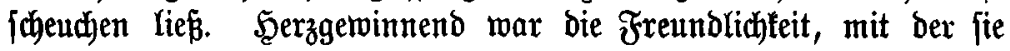
jeben Befuh empfing, bie warme Danfbarteit mit ber fie jeden $\mathfrak{B e r}=$ fuch ihr eine Frreube zu madhen belohnte.

So lebte Ehriftophine Reinward in ihrer befdjeibenen aber

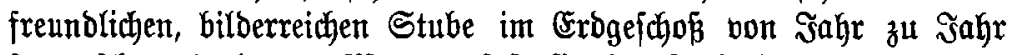

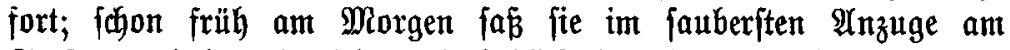
Fenfter, und hatte für jeben Şineinblidfenden einen freundliden (Gruß̌. Shre aud zuletgt nur wenig gebeugte (Gejtalt trug leine Spur greijen= 


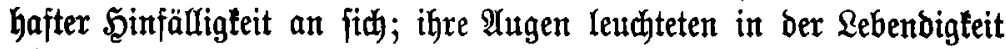
Des (Bejpräcts jugendlich, ihre Wange war zart geröthet; ihre Sprache,

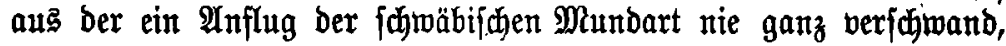

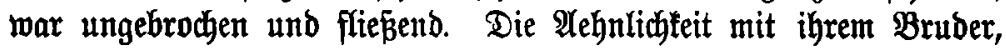
weldhe man an ber Lebenden nur wenig bemertte, trat in ber rubig faflummernden Reidje auffallento Gervor, bejonders in ber obern \$älfte Des Befididt.

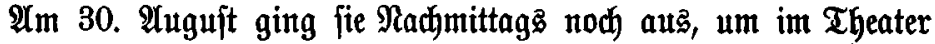
bie newe $\mathfrak{A r b e i t}$ eines $\mathfrak{M a l e r}$ zu jehen, an ber fie ihre voljte Theil= nahme zeigte. (Segen $\mathfrak{A b e n t}$ fühlte fie fich unwohl, meinte aber eines

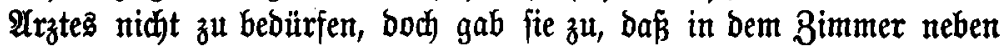
ihrer Salaffammer eine $\mathfrak{W a ̈ r t e r i n ~ b l i e b . ~ D i e f e ~ h o ̈ r t e ~ f i e ~ m e h r m a l s ~}$ über Samerzen Hagen, bann bas Baterumfer beten; als fie nad) längerer 将ufe gegen Mlorgen in bie Rammer trat, fanb fie bie (Sreifin mit gejollofienen alugen janft entfallafen.

So trönte ein jeligea (Ende ohne lörperliduen ober geiftigen $\mathfrak{B e r}=$ fall bas reidhe und glüfflidye Leben einer Frau, bie dem Sreife ihrer Freunbe merfwürbig und unvergę̧lid) fein würbe, aud wenn fie nidł Sdjiller's Sdjwejter gemejen märe. Irber fie war bie mürbige Sdywejter eines joldhen Brubers. Sie hat bie aud ihr befreundete Raroline von $\mathfrak{B o l z o g e n}$ nur um menige Monate überlebt, und wir fteben an ihrem Brabe mit bem Befühle, baß wir hier bie lek̨te Mittebende und Beugin einer gropenen und herrtichen Beit zux $\Re$ uhe bejtattet haben.

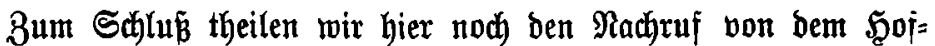
prebiger Dr. Afermann mit, aus bem. Meininger Bolłablatt 1847. $\mathfrak{N}$. 40:

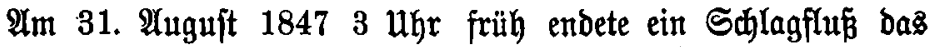

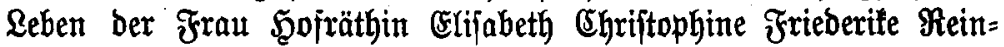
walb geb. Sdjiller wenige Tage vor ihrem 90;ten Beburtżtag; fein Srantenlager, nur ein vorübergehendes $\mathfrak{u}$ nwohlfenn war biejem $\mathbb{a r r}=$

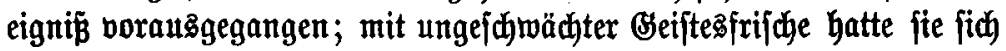

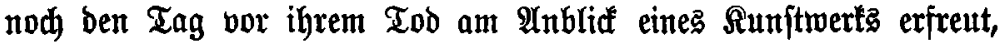
Das zur Bejdauung hier augejeftellt war; ihr Leben verflang, wie ez geführt morben sar, im Bejühl bes Schönen und Giuten, womit Brott bie Welt aużgejtattet hat. Alllgemein und innig war bie Theil= 
nahme, bie ihr Şillfdeiben herborrief; benn fie war allgemein verehrt und geliebt; in allen Ständen waren ihr bie Şerzen zugethan; unjre Serrfataften und bie verwandten und befreundeten BSfieber unfres

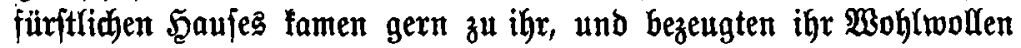
und Adtung; Durdareifende und Frembe fuditen fie auf, und fohieden mit angenehmen Eindrüdfen und Erinnerungen von ihr; Freunde und Freunbinnen gingen bei ind aus und ein, und erquidten fich an ihrem Umgang und an ihren Bsejprächen, und wie Biele, benen fie wohlgethan hatte, (pradjen ihr freundlid) grüßent zu, wenn fie an

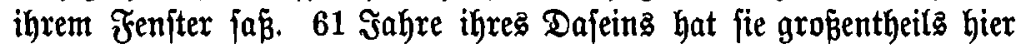

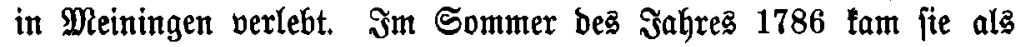
bie Battin des Bibliothefar's Reinwald, hieher; nadh bes Mannes $\mathfrak{T o b}$, Der im $\mathfrak{\Im a h y} 1815$ erfolgte, zog fie in ihr $\mathfrak{B a t e r l a m b}$ und lebte

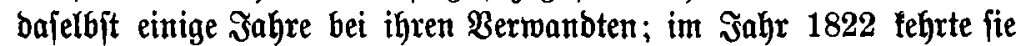
mit einer Freunbin, bie in Stuttgart zum Bejuch bei ihr gemejen wor, zurïßf, micthete fị bann päter bei biejer Freundin ein, und hat vom Sahr 1832 bis alt ihren Tod ununterbrodjen in ben ihr lieb geworbenen Räumen gewohnt.

$\mathscr{D} a \bar{B}$, was am meiften von ihr befannt war, and was fie für Biele bejonderz anziehend madyte, war vornebmlid zweierlei, einmal

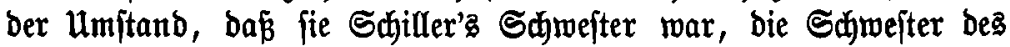

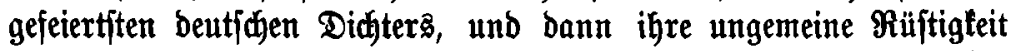

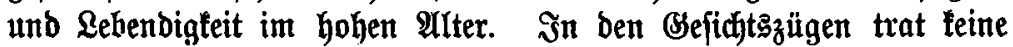

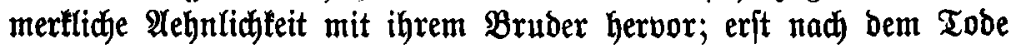
wollen Etlidje bas beuttidje Servortreten einer foldjen wahrgenommen haben. Defto unverfennbarer waren bie Büge ber $\mathfrak{A} e$ fnlichteit zwijden ihr und bem $\mathbf{B r u b e r}$ in ihrem Snnern. Sie hatte, wie er, ein be=

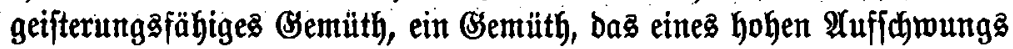
fähig wax; von allem geiftig und fittlich $\mathfrak{2}$ uzgezeidgneten füblte fie fíd) lebhaft ergriffen, uno (prach fidh leb̆haft barüber aus, und in ihrer $\mathfrak{A}$ ubobud bem Ermit und von ber $\mathfrak{B}$ ürbe bes Sdjillerifden Styl'g. Aud in $\mathfrak{A b}$ fidjt auf bidjterifde Begabung hatte fie etwas mit dem Brubar gemein; fie hatte aukerordentlid viel Sinn für Poefie und אumft; Sebidjte, Semälde, Runftanfdaaungen aller 9 rat waren ihr eine Lebensfreube und ein Rebenselement, - jie erquidte, fie erbaute fin baran, fie war Bott unb ben Mlenjđjen bantbar für joldje Bsenüffe. Shre frohen ober frommen Erregungen iđrieb fie nidyt felten in 
Berjen nieber, am liebften und am häufigiten aber befduäftigte fie fidd

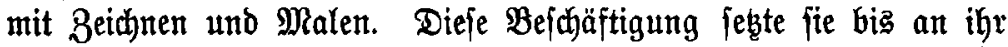
Enbe fort; nod) am lebeten Morgen ifres Lebenz hatte fie ein Frudt= und $\mathfrak{B l u m e n}$ [tǘf zu malen angefangen. $\mathfrak{A}$ ud in bem, was fie zeid)

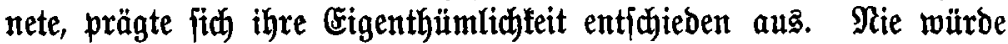
fie fin bazu verjtanden haben, eine Saritatur zu zeidnen. Sie zeid)= reète am Yiebiten $\mathfrak{B l u m e n}$ und Frühte, and diefe vorzüglich gern

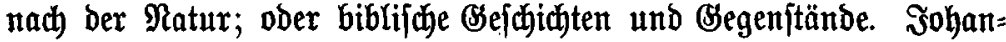

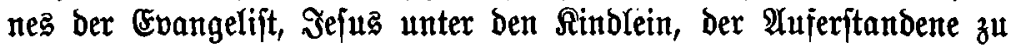
Emmaus, ben bie જ̛üngex am Brobbrecten erfennen, - bas maren Bilber, bie fie nidjt milde wurbe, theils zul copirent, theils felbit zu entwerfen. Mit ihren Beidfnungen und $\mathfrak{B}$ itbern idhmitute fie ihre Wohnung aub, ober bejuentte ihre Freunde und Freundinnen bamit.

Bater und Mautter ehrte fie lebenslänglich mit ber findlichjten Danfbarfeit. „Mein $\mathfrak{B a t e r}$, äukerte fie oft, war ein vortrefflidyer Mann; bei alfem, was idh vorbabe, frage id midy, ob er ez aud billigen witrbe." Wie für bie Êttern, fo hatte fie aud für bie (Bese=

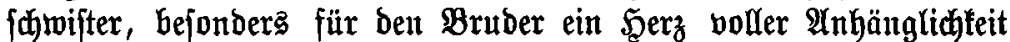

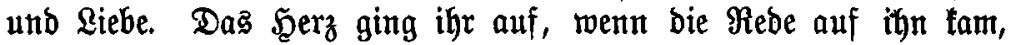
und wenn fie Diế und șents von ihm erzählte, waş in teiner Lebensbejdreibung von ihm jtand; wenn jie unbermuthet irgenbwo

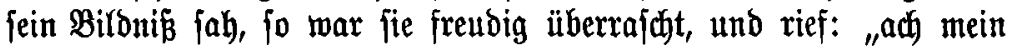
Bruder!" Yatber bei aller Berehrung und Qiebe für biejen Bruder war fie weit bavon entfernt, bieje Berefrung zur Sdja zu tragen,

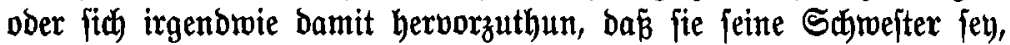
oder von ihrem Bruder und bon feinen $\mathfrak{B e r k e n ~ m i t ~ a l l e r ~} \mathfrak{B e l t}$ zu ppredjen; von freien Stüdén pprad fie nidgt leidgt über ihn; wer fie nidht ausbrürfflich auf biejen Begenjtanb brachte, hörte und exfuhr Darüber nidfte von ihr.

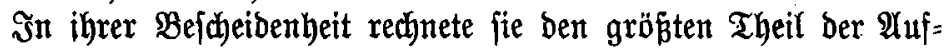
merffamfeit und âtatung, bie man ihr berwies, nicht fid , fonbern ihrem Familiennamen und ihrem Berhältniß̄ zu bem Bruber zu.

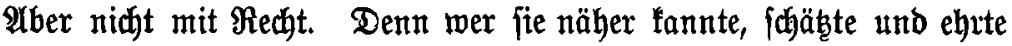
fie um ihrer jelbft wilfen, und ganz abgefehen von ber bejprochenen Beżiehung. $\mathfrak{B a z}$ zunädft bei ihn in bie $\mathfrak{A}$ ugen fiel, uno ant häu= figiten erwähnt unb bewundert wurbe, war ihre Rebensfräjtigfeit; fie war eine burchaus fernige Statur, bie lek̨ten Sahrzehnte fđjienen fpurlos an ihr vorüber gegangen zu jenn; jelbjt von bem heftigen 
Sranfbeiţanfall, ben fie mehrere Sahre vor ihrem Tod zu bejtehen hatte, erholte fie jidh zum Berwunbern fanell, und ohne alle Spur

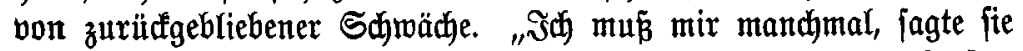
nidjt felten ladjend, ben ganzen Snghalt meines Rebens zurüdfrufen,

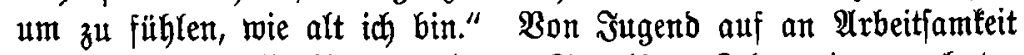
gewöhnt, blieb fie bis zum leşten Tag igres Rebeñ in getwohnter Beife thätig; fie hatte nur eine $\mathfrak{a b}=$ und zugehende $\mathfrak{B}$ edienung; bas Meijte, was in andern Scäujern burd) Dienjtboten gefdieht, that fie

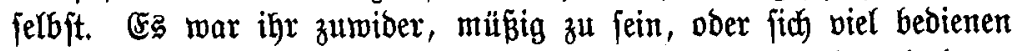
zu la fjen, ober bas, wą fie gern raja angriff, zauberno und lang= fam vornehmen z̆ jehn. "Das erbält munter und jrifh, und ver= treibt bie \&angeweite," erwieberte fte fijerzend, went ihr eine -Min= berung ihrer häualidaen Gsefdäftigfeit anempfofhen wurbe. Sie hätte fich mandbe Bremächlichleit ver[dhaffen, ihr Dafenn und ihre Ulmgebung mit mandjer Bequemlidłeit und $\mathfrak{A} n$ nehmlidbeit ausftatten fönnen; bie Mittel murben ihr bazu geboten; ihren $\mathfrak{B e r w a n d t e n , ~ b i e ~ f i e ~ h e r z = ~}$ (id) riebten, wie unjerm Eandesherrn, ber ihre ßenfion auf bas Doppelte erhöht hatte, wäre es Freube gewefen, ihr in biefen $\mathfrak{B e}=$

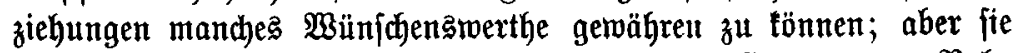

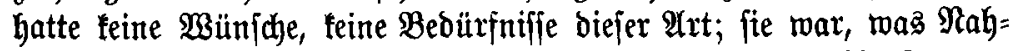

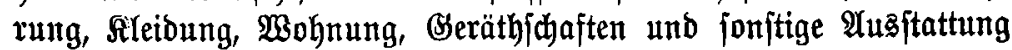

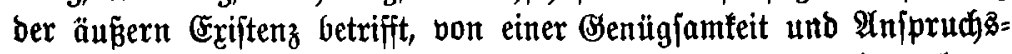
lojigfeit, wie fie bent zu Tage, jelbjt in geringeren Ständen, faum mehr gefunden wirb, ja man mögte jagen wegen ber ganz andern Einbrücfe und Bsemöhnungen, unter benen bie Jugend ję̧t groß wirb, faum mehr möglich ift. (Sinfachbeit, pruntlofe (Einfachleit wax ein Brundzug ihres wejens und ihres \&ebens. 2ulfes. Heberflüfitige, Ueppige, Berweidhlichende, Luxuriöfe mogte fie nicht, und hielt es fern von jidd; und ihren Begriffen nach war Bieles überflüffig und vermöhnend, mas $\mathfrak{A}$ ndern für ganz unentbehrlidy galt. Mit nied=lidhen Speifen und Geridyten war ihr nidt gebient; bie einfadjite Sojt war ihr bie liebjte, felbjt nach einer überitandenen Rrantheit,

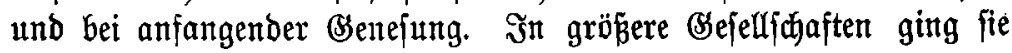

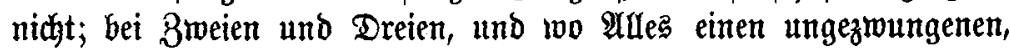

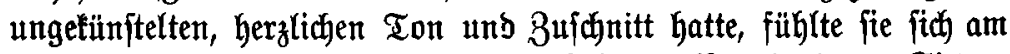
wohliten. Dem Bolf, und allen ben Rebenatreijen, in benen Sitten=

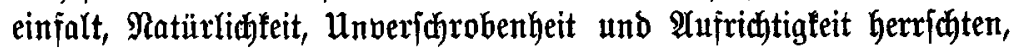
war fie von ganzer Seele zugethan. „Mir ift wohl, fteht in ihrem 
Tagebuch, unter euch Menjijen von nieberem Stande. Da findet

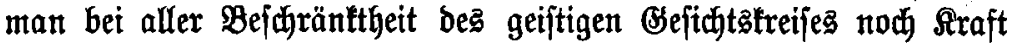
und Selbftitänbigteit. Und was ît unire A̛fflärung, unjre Bilbung?

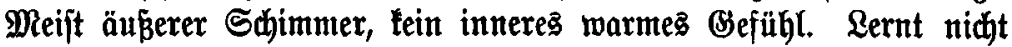
felbft bie heilige Sprache ber Seele, bie Empfindung, eine frembe Sprache, und wirb fie nidyt oft burd Conpenienz und Dredjfelei zur $\mathfrak{U}$ nnatur?"

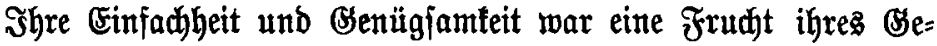
mütha, und ihrer Erziehung. Unter Entbehrungen und Berjagungen von manderlei $\mathfrak{A}$ rt war fie aufgemadfien im elterlidjen $\mathfrak{S a \mathfrak { a }}$; ein Sleib von felbit gepponnenem Beug, eine Frubt von bem Baum, ben Des Baters Seand gepflanzt und gepflegt hatte, - bas maren in ihrer Jugend Freuben und Baben von bedeutendem $\mathfrak{B e r t h}$ für fie. Wie fehr lam ihr eine Derartige Sngend und eine foldhe Sinnesmeife im fpäteren Leben za Sute! Shr Gatte, ein jehr braver und hod)= geađteter Mann, aber tränflich und zum Trübjinn geneigt, hatte nidgt mehr als 300 Sulden Bejoldung, und von bem, waz zur Scaußhaltung und Lebensführung gehört, und wả zur Erheiterung und Beglüdưng einer jungen Frau gereidhen fann, wapte er, ber fajt $\mathfrak{n u r}$ in feinen $B$ üdaern und in feiner Bjelebrjamfeit lebte, wenig

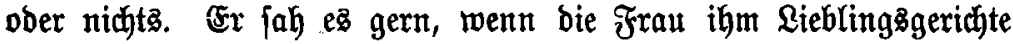

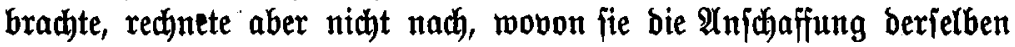
beftritt, erfannte nidht, Dás fie fich jelbjt alle fie für ibn erübrigte, und verjagte ihr nidht felten tleine Summen, Die fie blos zu bem 3wedf, um größere 2 Hagaben zu exjparen, von ibm verlangte, intem fie Bieles, was im Scaule notfig war, felbjt verfertigen wollte. Bon Bejuthen war er fein Freund, jelbjt Frauen hielt er vom $\mathfrak{u m g a n g}$ mit ber jeinigen möglidjit fern; nicht autz Mangel an Liebe für feine Frau, oder weil er ihr teine Freube ge=

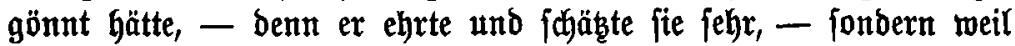
ex glaubte, einen Belehrten und einen Didter zum Manne zu haben, bas reidje für fỉd idjon hin, eine Battin zu beglücten. In fo ein=

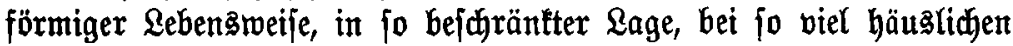
Entbehrungen, Ânftrengungen, Dpfern, bei fo wenig $\mathfrak{A}$ ufheiterung und

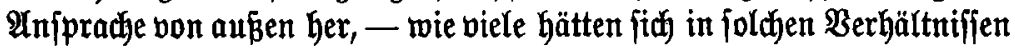

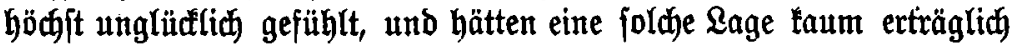
gefunden! Midut jo unjre Bollendete! Man hörte leine Silage von

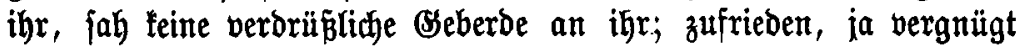


ging fie ihren ftillen (Sang burd's \&eben. "Bsie wohl fühle ich mid in meinem fleinen Reben, zeidhnete fie in ihr Tagebudy auf, und befeligt burd eine höhere Mlacht, bie inz tieffte Şerz blicft, lerne id in biejem bejdeibnen Sopje ein hohes Briüd finden." Forjden wir

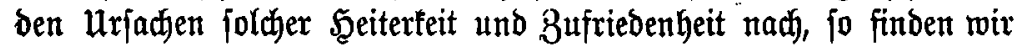
bieje zum Theil in ber idon vorhin erwähnten Gerwöhnung zur Einfachbeit und $\mathfrak{A n}$ prudblofigteit von Jugend auf; zum Theil lagen fie in ifrer gefunden fräftigen Natur, bie nichts đrämlichea, und feine tranthaften Berftimmungen auffommen Ließ ; zum Theil waren

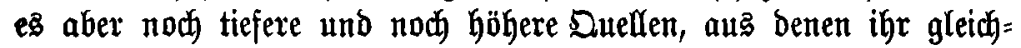
mäß̈tg frifłer und fröhlicher Sinn entiprang. Sie hatte eine $\mathfrak{B a h r}=$

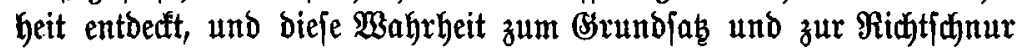
für fich erfoben, bie für bie Mehrzahl ber Jekttlebenden in ferner Berborgenheit zu liegen \{hjeint; Biele der Jekttlebenden bringen ebs nidht einmal bis zur Erfenntnif biefer Wahrheit; nod Wentigern gelingt bie mirflithe Exfajiung berjelben, und ihre (Finfübrung in ihr Leben. Denn unjer Sinn ijt überwiegend auj ben Reiz, auj ben Medffel, auf bie Füllfe geridhtet; unz graut, uns bangt vor ge= nußjleeren Tagen, ja nicht jelten vor genußjleeren Stunden; auf ge= wohnte Ânnehmlithleeiten zu verzichten, halten wir für thörigt, und bedauern ber, ben bie Umitände, oder;gewiffie Trauerfälle bazu nöthigen. Tie Selige aber badyte und fprach: ,aud auts ber (Ent= fagung quellen Freuben!" - und gewiß̄ ! venn irgenowo, fo hat

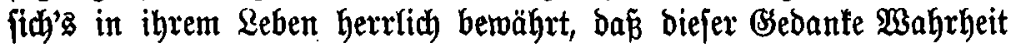
ift. Die Beridbte ber Reifenden erzählen bon fandigen Segenden im nörbliden âtrifa, bie reid an verborgenen Duellen finto. Buräbt man bier einige $\mathfrak{F} u \mathfrak{p}$ tief ein, und gräbt eine foldhe Quelle auf, fo wanbelt fich bie Natur bes Bobens in turzer Beit auf zauberifhe Beife um. Frijhes erquidfendes Srün dringt ba herbor, wo vorher nidjts als glühender Fflugjand lag, und Blumen und Frübte ent= fprofjen bem Boben, ber zubor alles berjagen zu wollen jojien. $\mathfrak{A} u \tilde{f}$

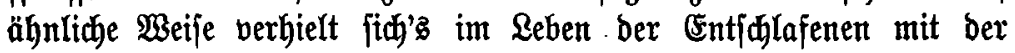

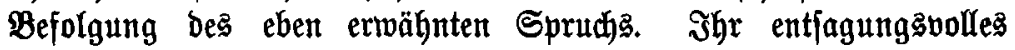
seben faien Mandien ein öbes, freubenleeres Reben zu fenn. Sin ber That aber war es bas Gegentheil. Denn, - jebetent ihre Ent= jagungen fie nicht in ben Stand, Andern Freube .au jafaffen, Freube zu bereiten? gemährten fie ihr nidjt bie Mittel, wohlzuthun und mitzuttheilen? (F) ift auperordentlid, wie Biel (sutes bie Beremigte 
im Stillen gethan hat, - und bei allen Bselegenbeiten, wo es einen eblen und fhönen 3meă zu förbern galt, war fie unter ben willigen und fröhliduen Sebern ftets voran. Sie ftridte Strümpfe, fie fertigte Rleibungajtüufe für bie $\mathfrak{A r m e n}$, und wie freute jie jid, wenn ber

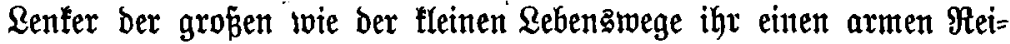
jenden zuführte, ber ihr mit wahrem $\mathfrak{B o h l g e f u ̈ h l ~ f u ̈ r ~ b i e ~ m a r m e ~}$

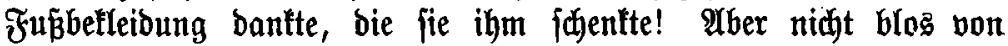

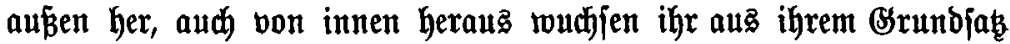
von ber Entfagung Freuden und Gsenüffe zu. Denn biejer Brand=

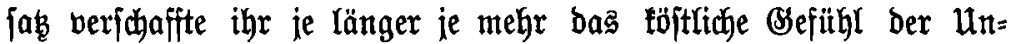
abhängigfeit, ber innern $\mathfrak{H n a b h a ̈ n g i g f e i t ~ u n d ~ F r e i h e i t ; ~ e r ~ v e r f đ a f f t e ~}$

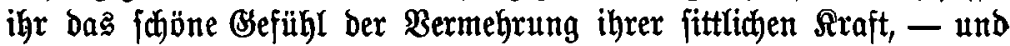
vor allen Dingen bewahrte er ihr Gsemüth vor bem, was bie unfrigen

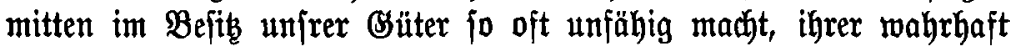
froh zu werben, - er berwahrte fie vor $\mathfrak{H e b e r j a ̈ t t i g u n g ~ u n d ~} \mathfrak{A} b=$

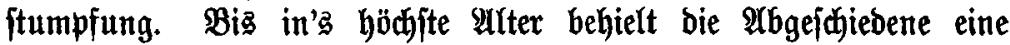

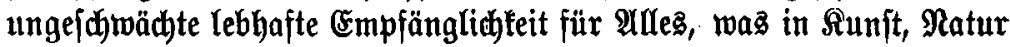
und Menjhentwelt 2lnjprechendes fidh ihr bot; aud über Seringez tonnte fie fid fo herzlich und fo innig freuen, wie Andere es faum über Bebeutendes und Grobes fönnen; Das hätte jie nidgt gefonnt, wenn niđat Mäp̃igung und Entjagung bie Bsefährtinnen ihrer ługend und iłrę 2 ebens gewwefen wären!

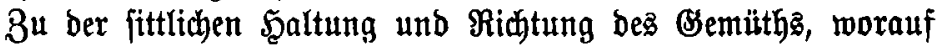
ber frohe und zufriebene Rebenşinn ber Bollendeten hauptjächlid be= rubte, gefellte fich bei ihr echte Frömmigfeit alg Brundjtein und Pfeiler für ben ganzen $\mathfrak{A}$ ufbau ihres Denfens, Wolfenz und Thuns hinzu. Fhłe Frömmigfeit war burchaus naturrwübjig und gefund; ez war nichts (Gemadjtes, Bsetüniteltes, Eramungenes babei; - teine

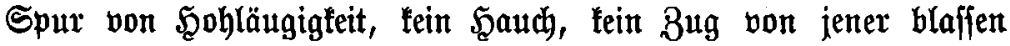
thränenjeligen $\mathfrak{A n b a d j t , ~ b i e ~ e s ~ n i d j t ~ b e g r e i f e n ~ t a n n , ~ w a r u m ~ b e r ~ S i m = ~}$ mel iiber ber fündentollen crobe fo freundlid ift. Şodherzig wie iht ganzez Bejen, jo wax audi ihr Botteşglaube; jie verjtand, fie

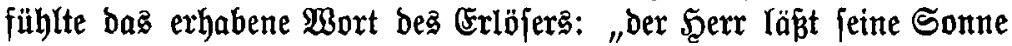
aufgehen über bie Biuten und über bie $\mathfrak{B o ̈ j e n " ~ - ~ f e i n e r ~ g a n z e n ~}$ (Sröße nad); ihr Gott war nidyt ber Bott bes Gifers, fonbern ber

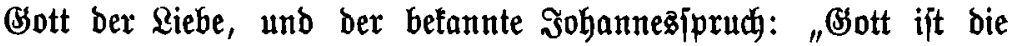

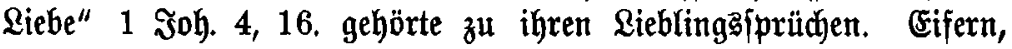
fich) ereifern, fich lebhaft ereifern, - bas tonnte unfre Seeimgegangene 
auth; fie war feinesiwegs eine von dent marklojen Raturen, Denen e? non $\mathfrak{S a u s}$ aus an aller 3ornmüthigleit gebridyt. Im Segentheil!

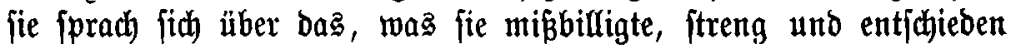

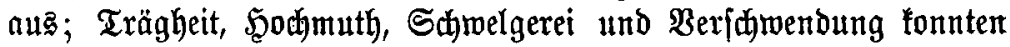
fie in lebhafte Entruiftung verjesen, ia fie wurbe Feuer uno Flamme, wenn empörende Ungeredytigfeiten zur Sprache fanten; ober wenn fie gegen bas \&after ber $\mathfrak{B o l u h i t , ~ b e m ~ f i e ~ a m ~ m e i j t e n ~ g r a m ~ w a r , ~ z u ~}$ Felbe zog. Galle biefe Entrüitungen und Ereiferungen gingen aber

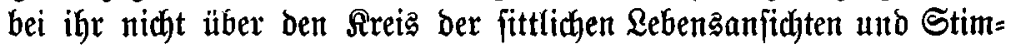
nungen hinaus; auf ihre religiöfen $\mathfrak{U n}$ ftudten, auj ihre religiöjen (Befühle wirtten fie nidht ftörend und trübend ein, BSott und bas Bättliche jtanden ihr hoch über Den Reibenjhaften unt Berirrungen

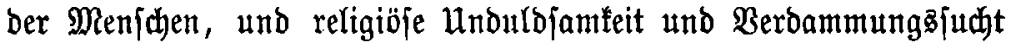
fanden feinen $\mathfrak{A n t l a n g}$ in ihrer Seele. Es geht ein Borurtheil butrch

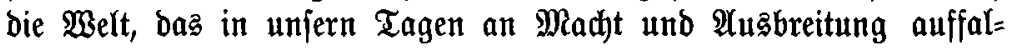
Yend zunimmt, und fid mit Erfolg bentüht, bem jestetebenden Ge= (a)lecht alle Frömmigleit grünblich zu verleiben. Das ift bas $\mathfrak{B o r}=$ urtheil: Frömmigfeit vertrage fidh nid)t mit Fröhlidfteit; bie Fröb= lichfeit müfffe ber Frömmigłeit weichen, ja bie Frömmigłeit mache ber Fröblidbleit Selbjtwernichtung zur ßflicht. Sft ein Reben geeignet, bię̧ Borurtheil zu wiberlegen und feine gänzliche Brundofigfeit ïberzengend barzuthun, fo ift es bas \&eben, bas als ein fröhlid und felig vollentbetes vor unz liegt. S̃lt ber That! bei unferer Bertlärten ging beibes, bas Frommpein und bas Fröblidjein ftets Şand in Şand, wie ein GSejdyijterpaar; fie vertrugen fich nicht blog vortreff= (ich) mit einander, fie soirtten auch förbernb auf einander ein; fie unterjtübten, erhähten und veridjönerten einander, und das alfes ohne befondern 9 lufwand von Mähe, fonbern fo natürlid und fo von felbft, wie Lerchengefang und Frühlingąhimmel. Die glüd =

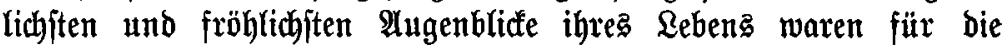
Seimgegangene bie, in benen jie fich innerlich gebrungen fühlte, Dem Iteben Gott zu banfen. Ulnd wie oft fühlte fie Beranlaffung bazu! benn jebe gute Brabe, bie fie hieniedent empfing, regte fromme Dant= gefühle in ihr an. "(Sott lyat mid wunberbar und fegensareich ge= führt!" - das war ihre bejtändige Bertündigung; man barf fie in

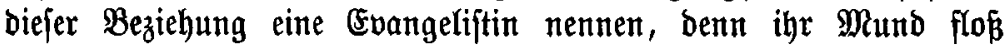
allezeit über von dem, wovon ihr šerz voll war, von ber Gäte (Sottes, bie fie ifrent (Sefühl nach in fo reichem Mape an fich er= 


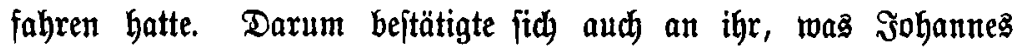
fajreibt: "bie völlige Riebe treibt bie Furdtt aus." 1 ฐoh. 4, 18. $\mathfrak{D a} \bar{g}$, was un âfe mehr oder minder beunruhigt und quält, was oft fo bujfter und riejengroß aufiteigt in unz, und feinen falten Sihatten weit hinaużwirft auf bie fonnige Flur unjer Lebenż, -

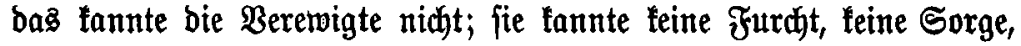
feine Bangigłeit vor ber Bufunft. "(Bott hat midy ja fo lieb! warum jollte ex mir Hebles thun?" äuperte fie, wenn von foblimmen Mög= lichteiten auf ängftliche Weife gerebet wurbe. Fhre Frömmigteit wax, was fie fein foll, und wie fie in ber Sharift genannt mirb! Bott= jeligfeit!

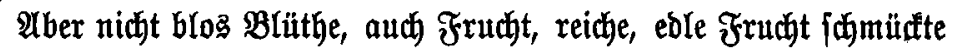
ben $\mathfrak{B a u m}$ ber Frömmigfeit bei ber Dahingegangenen. Sie fah ihar Leben an wie einen Botteabienjt; mehr nod), fie führte es wie einen joldjen. Arme Menjujen auf Eroen, meinte fie, feien Werlzeuge Bot= tea, und es fen ihr Brfürt wie ihre Eyre, basjenige zu erfennen und auşzuridjten, was sott für jie bejtimmt habe, und was er burch fie auşgeridgtet haben wolle. Betrachtungen und Erwägungen biejer

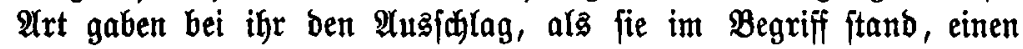
ber widhtigften Sdaritte ihres Lebens zn thun. (E) ift aus Shiller's

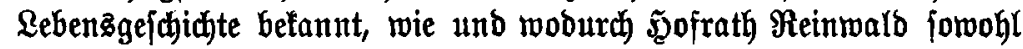
mit bem Didjter, als audy mit befien Sdjwejter in Berührung und

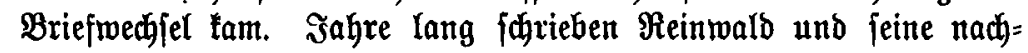
herige Battin an einander, ohne bã

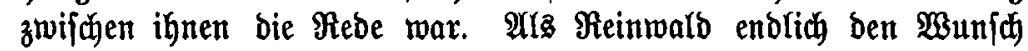
einer foldhen ausgejprodfen, und um bie Şand jeiner Correjpondentin angehalten hatte, wendete fid biele, ehe fie bas Sawort gab, an ben

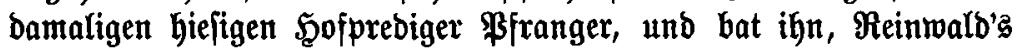
genaueften Freund, um feinen $\Re a t h$. Pfranger antwortete ihr: "aus

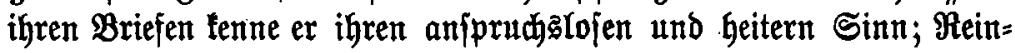
walb fey tränflich und habe $\mathfrak{A}$ nlage zur Şgpodjondrie; beshalb ftehe er ziemlich einfam und freublos in ber $\mathfrak{B}$ elt, und es würbe baz

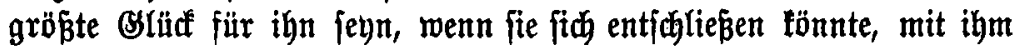

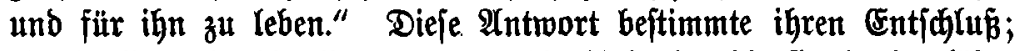
bie Stellung in bie fie eintreten, bie $\mathfrak{A} u f g a b e$, bie fie barin löfent

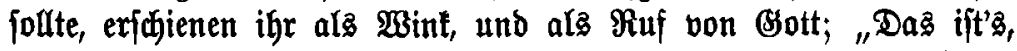
Dadjte fie, twas Bstt in biejem Leben von Dir gethan und geleiftet haben will; was Du hienieden Bjutes zu thun iduldoig bift, folfit 
Du an biejem Manne thun!" Run jagte fie freubig $\mathfrak{a} a,-$ und nie in ihrem Reben hat fie ihre $\mathfrak{A} n$ fitgt über biefen \$unlt geändert; es ging ihr nicht wie benen, bie fith in aufgeregter Stimmung einent Berufe miomen, ben fie nadhher, ment bie $\mathfrak{A}$ ufregung vorüber ift, boch nidbt für ben ihrigen erfennen. Im Slauben, im freubigen BSlauben, wie jie ben neuen \&ebensweg angetreten hatte, fo wandelte fie fort und fort auf ihm, fo vollendete fie ihn, atlo now am Sdjlup

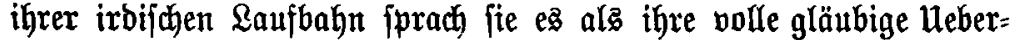

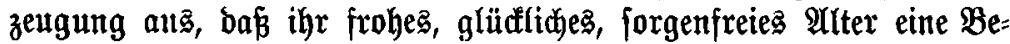
lohnung und eine Beftätigung von Bott fey; bie Beftätigung, Da fie jeinen Millen veritanden und befolgt habe, als fie fith zum $\mathfrak{W e g}=$

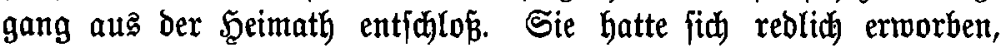

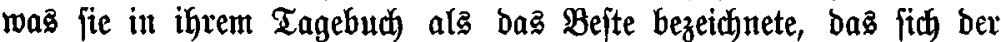
Menjh in biefem Leben erwerben tönne, "nemlich ein rubiges $3 \mathfrak{u}=$ rüffichauen auf bie Bergangenheit;" fie mar ber Miahnung und Fror= berung treutidi nadigetontmen, bie fie in poetijder Frorm in ihre Erinnerungablätter niebergelegt hatte, und bie eine jprectende $\mathfrak{a} \mathfrak{b}$ fpiegelung ihrer ganzen (Eigenthümlid)teit enthalten:
\end{abstract}

wandle nur treu vor bem Şerrn, und führe ein heiliges Leben; nimm Heinen Theil am Bseifte ber Beit, ex reift zum Beridjte!

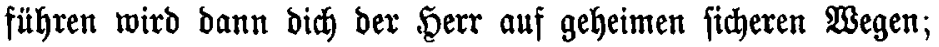
alles bejikejt bu erwig wenn bu nur alles igm weiheft; audh bein Scherflein im Silauben gebracht ijt töftliche Babe, gieb ihm alles mit Freuben, behalte nidte als bie Riebe!

Eine Frömmigteit, wie bie ber Bolfendeten war, läpt fich nicht burch Ueberlegung und Borjak zu Stande bringen; fie tommt auch nidft blos Durd Unterridft und $B$ üdher in bas Seerz hinein, weder

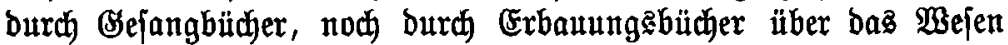

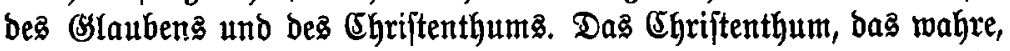
fernige, Yebenbige (Shriftenthum ift und hat Durdhaus etroa unmit=

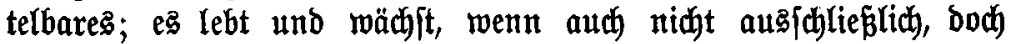
vorzugämeife, wie bie wahre ßoefie, vom Sinntichen und unnill=

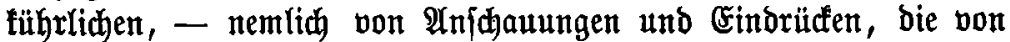
felbjt in's snnere bringen. Die Frömmigteit, bie von Rinoheit auf jo zu jagen eingeathmet wirb, - bie Frömmigfeit, bie bas sino im

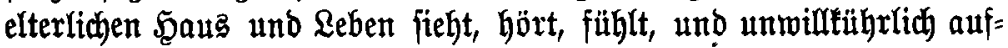
nimmt, - bie wurzelt am tiefiten und fejteften, weit fefter, als bie, 
bie man bem Menjuen gefliffentlid beizubringen jucht, und beren Werth und $\mathfrak{u n e n t b e h r l i d f e e i t ~ m a n ~ i h m ~ a u f ~ e i n l e u d t e n d e ~}$ Beife aus= einanderjekt; benn bas Eindringen jener ijt nidjt fein $\mathfrak{W e r f ,}$ wohl

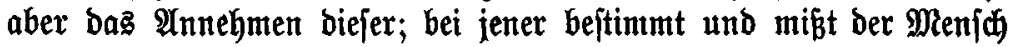
nidft ab, wie viel fie ihm gelten foll; wohl aber bei biejer; bieje hat nur jo viel Macht über ihn, als er ihr zuerfennen und zugeftehen will, - und wo bas (Söttlidje bem menjulliden $\mathfrak{u}$ rtheil mehr ober minbex unterthan wirb, ba ift es eben bas wahrhaft sö̈ttlidge nicht mehr.

Unjire Entidhlajene wudbs in frommer $\mathfrak{u m g e b u n g , ~ u n d ~ u n t e r ~}$ frommen Eindrüden unb Gintwirtungen auf, mie bentn überhaupt igr

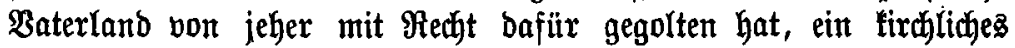

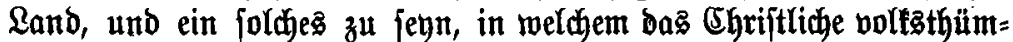
lich gemorben, und mit der häuglichen Sitte innig verjdmolzen ift. Shjon oben wurbe ermäbnt, wie lieb der Bollendeten baz ojter= ebangelium von Den Wanbrern nadh Emmaus war. Dieje Riebe beruhte hauptjählich auf einem ihr unvergę̧lichen cindruct aus ben Rinberjahren. "Einjt, - fo erzählte fite biejen Borgang, - ba wir als Rinber mit ber lieben Mutter zu ben Gropeltern gingen, nahm

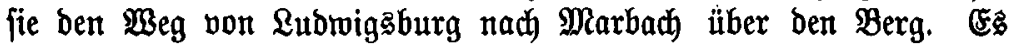

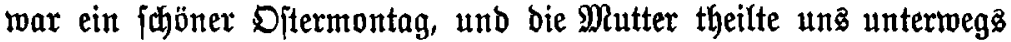
bie Shejdidjte von ben zwei Süngern mit, Denen fich auf ihrer Ban=

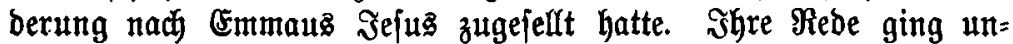

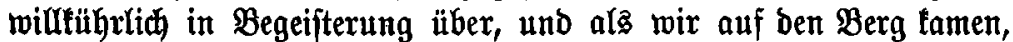
waren wix jo gerührt, baß wir niebertnieten uno beteten. Diejer $\mathfrak{B e r g}$ wurbe uns zum Tabor!"

So tief innerlich bergleidhen Cinbrüafe und Erlebniffe für bie Seimgegangene gewejen waren, fo tief innerlid) pflegte fie biejelben aud zul halten; fie trug fie nidht auf ben Rippen, mifate fie nidft als $\mathfrak{B u ̈ r z e}$ in bie $\mathfrak{u n t e r f}$ altung ein, langte fie nidht hervor, um fid

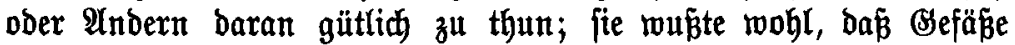
mit föftlicher Marbe fühl gehalten fein wollen, fonjt verbuften fie! -

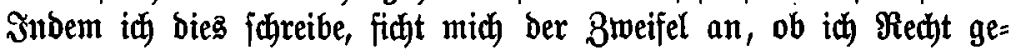
than habe, bieje Züge aus bem Lebenzbild ber (Entjhlafenen zu ent= werfen und ber Deffenttlidfeit zu übergeben? A̛tles $\mathfrak{A} \mathfrak{u f f e h e n ~ m a c h e n , ~}$ und alles geipreizte prunfende Wejen war bem idjlidjten gejunden Sinn ber Bollendeten ganz und gar zumider, und S(ameidfler ver= bienten fid mahrlid) feinen Danf bei ihx. $\mathfrak{3}$ ürbe fie es baher wohl 


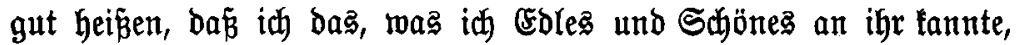
hier an bas Lidjt ftelle? Würbe fie zufrieben fegn mit bem Billo, was id hier von ihr gebe, ober wïrbe fie nidyt viefleidft, wenn fie ez jähe, unwillig auj mid bliden and fagen: "Du hajt mit zu glänzenden Farben gemalt, uno wo ift denn ber Sdjatten? Bin

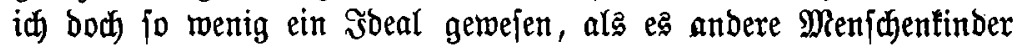
find, uno hab' ę aud gar nidjt feyn wollen. Went etwas (Sutes an mix gewejent ift, jo ijt es meine gute Ratur, - Bottes Babe!"

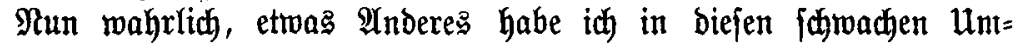
riffen eigentlidh nidht barjtellen und augführen wollen, als bieß̃: jeht!

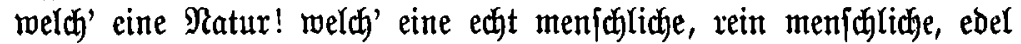
meniflidfe Natur! und wie wohlthuend, wie erfrifdjend, wie erhebend

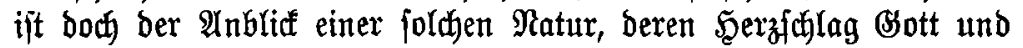
bie \&iebe ift - - habe id bies nidgt erreidft, bringt meine Stizze eiuen andern cinbruff als biejen hernor, jo bitte id bie Shald Dabon nicht meinem berzen und feiner Billenameinung, fondern meiner ,̧and und meiner Feber zuzufdreiben. 
